\title{
On the General Hodge Conjecture for Abelian Varieties of CM-type
}

By

Fumio HAZAMA*

\begin{abstract}
The General Hodge Conjecture for abelian varieties of CM-type is shown to be implied by the usual Hodge Conjecture for those up to codimension two.
\end{abstract}

\section{$\S 1 . \quad$ Introduction}

The purpose of this article is to show that the validity of the Hodge Conjecture in codimension two implies that of the whole General Hodge Conjecture (GHC for short) for any abelian varieties of CM-type. The main ingredient is the theory of abelian varieties associated to hyperplane arrangements as is developed in [3]. In particular, the notion of " $N$-dominatedness" introduced in [3] plays an essential role for us to understand what kind of exceptional Hodge cycles should be proved to be algebraic. Our strategy for the proof goes roughly as follows. Given a Galois CM-field $K$ with $\operatorname{Gal}(K / \mathbf{Q}) \cong G$, we associate an abelian variety $A_{\boldsymbol{A}\left(2^{n}\right)}(G ; K)$ to a hyperplane arrangement $\boldsymbol{A}\left(2^{n}\right)$ in $\mathbf{R}^{n}$ (see Section five for the definition of $\boldsymbol{A}\left(2^{n}\right)$ ). Thereafter we show an arbitrary abelian variety $A$ of CM-type split by $K$ can be embedded into an appropriate self-product $A_{\boldsymbol{A}\left(2^{n}\right)}(G ; K)^{m}$ (Proposition 6.5). Thus GHC for $A$ is reduced to $\mathrm{GHC}$ for $A_{\boldsymbol{A}\left(2^{n}\right)}(G ; K)^{m}$ (Lemma 2.1). Furthermore we reduce GHC for $A_{\boldsymbol{A}\left(2^{n}\right)}(G ; K)^{m}$ to the usual Hodge Conjecture for it by translating the properties of various rational sub-Hodge structures of its cohomology spaces

Communicated by K. Saito. Received February 25, 2002. Revised September 5, 2002.

2000 Mathematics Subject Classification(s): 14C30

Key words: Hodge Conjecture, abelian variety, hyperplane arrangement

*Department of Natural Sciences, College of Science and Engineering, Tokyo Denki University, Hatoyama, Saitama, 350-0394, Japan.

Fax number: 049-296-5132 
into some combinatorial properties of the arrangement $\boldsymbol{A}\left(2^{n}\right)$. Thus the fact that $A_{\boldsymbol{A}\left(2^{n}\right)}(G ; K)$ is 2-dominated (Theorem 7.14) implies the aforementioned result.

The plan of this paper is as follows. In Section two we recall the formulation of GHC and prove some auxiliary results. In Section three we recall the definition and some properties of the $A V$-matrix associated in [3] to hyperplane arrangements. Thereafter we introduce a matrix, called Hodge matrix, the kernel of which reflects the structure of the ring of Hodge cycles on a certain family of abelian varieties of CM-type. In Section four we recall some general facts about abelian varieties of CM-type and their Hodge rings. Here we introduce the notion of $h$-degeneracy, which will play an important role when we investigate the structure of Hodge rings of various abelian varieties. In Section five we introduce the notion of CM-arrangement and explain how to associate an abelian variety of CM-type to an arbitrary CM-arrangement. At the end of this section, we introduce a hyperplane arrangement $\boldsymbol{A}\left(2^{n}\right)$, called that of $(2, \ldots, 2)$-type , which play an important role throughout this paper. In Section six we show that any abelian variety of CM-type is realized up to isogeny as an abelian subvariety of an abelian variety associated to the CM-arrangement of $(2, \ldots, 2)$-type. In Section seven we investigate the structure of the kernel of the Hodge matrix associated to the hyperplane arrangement $\boldsymbol{A}\left(2^{n}\right)$. As a result we will see that for any pair $(G, K)$, the abelian variety $A_{\boldsymbol{A}\left(2^{n}\right)}(G ; K)$ is 2-dominated in the sense of [3]. In Section eight we show that if Hodge cycles of codimension two on the abelian varieties of $(2, \ldots, 2)$-type are algebraic, then the whole GHC holds for them. As a result we obtain the validity of GHC for arbitrary abelian varieties of CM-type under the same hypothesis.

The author is grateful to the referee for the careful reading of the manuscript and his or her helpful suggestions for improving the paper.

\section{§2. General Hodge Conjecture}

In this section we recall the formulation of the General Hodge Conjecture, and prove some key lemmas.

Let $X$ be a smooth projective variety over $\mathbf{C}$. The arithmetic filtration $\left\{F_{a}^{p} H^{k}(X, \mathbf{Q})\right\}_{p \geq 0}$ is defined by

$F_{a}^{p} H^{k}(X, \mathbf{Q})=\left\{\gamma \in H^{k}(X, \mathbf{Q}) ; \gamma \in \operatorname{ker} i^{*}: H^{k}(X, \mathbf{Q}) \rightarrow H^{k}(X-Y, \mathbf{Q})\right.$

for some subvariety $Y \subset X$ of pure codimension $q \geq p$ in $X$ \}.

It is known that the kernel $\operatorname{ker} i^{*}: H^{k}(X, \mathbf{Q}) \rightarrow H^{k}(X-Y, \mathbf{Q})$ is always a rational sub-Hodge structure of $H^{k}(X, \mathbf{Q})$, and that $F_{a}^{p} H^{k}(X, \mathbf{Q})$ is always 
contained in $F^{p} H^{k}(X, \mathbf{C}) \cap H^{k}(X, \mathbf{Q})$, where $F^{p}$ denotes the Hodge filtration. Let

$$
\begin{aligned}
F_{h}^{p} H^{k}(X, \mathbf{Q})= & \text { the largest rational sub-Hodge structure } \\
& \text { in } F^{p} H^{k}(X, \mathbf{C}) \cap H^{k}(X, \mathbf{Q}) .
\end{aligned}
$$

Then the Grothendieck amended General Hodge Conjecture (GHC) is stated as follows:

$$
\operatorname{GHC}(p, k, X): F_{a}^{p} H^{k}(X, \mathbf{Q})=F_{h}^{p} H^{k}(X, \mathbf{Q}) .
$$

Another equivalent formulation of GHC uses the concept of level. For any rational sub-Hodge structure $W \subset H^{k}(X, \mathbf{Q})$, we define the level $\ell(W)$ by

$$
\ell(W)=\max \left\{p-q ; W_{\mathbf{C}}^{p, q} \neq 0\right\} .
$$

Then GHC for $X$ is stated as follows.

For any rational sub-Hodge structure

$W \subset H^{k}(X, \mathbf{Q})$ with $\ell(W)=k-2 p$, there exists

a Zariski-closed subset $Z$ of codimension $p$ on $X$ such that $W \subset \operatorname{ker}\left\{H^{k}(X, \mathbf{Q}) \rightarrow H^{k}(X-Z, \mathbf{Q})\right\}$.

We prove some lemmas for later use.

Lemma 2.1. $\quad$ Let $A$ be an abelian variety and $B$ an abelian subvariety of $A$. Then $G H C$ for $A$ implies that for $B$.

Proof of Lemma 2.1. One can easily check that if GHC holds for an abelian variety, then it also holds for any abelian variety isogenous to it. Hence by Poincare reducibility we may assume that there exists an abelian subvariety $B^{\prime}$ of $A$ such that $A=B \times B^{\prime}$. Let $\pi: A \rightarrow B$ denote the natural projection and $\iota: B \rightarrow A$ the natural inclusion. Let $\gamma \in F_{h}^{p} H^{k}(B, \mathbf{Q})$ for some $p, k$. Then $\pi^{*}(\gamma) \in F_{h}^{p} H^{k}(A, \mathbf{Q})$ by the functoriality of the Hodge filtration. Since GHC for $A$ is assumed to hold, we have $\pi^{*}(\gamma) \in F_{a}^{p} H^{k}(A, \mathbf{Q})$, hence there exists a subvariety $Y \subset A$ of pure codimension $q \geq p$ in $A$ such that

$$
\pi^{*}(\gamma) \in \operatorname{ker} i^{*}: H^{k}(A, \mathbf{Q}) \rightarrow H^{k}(A-Y, \mathbf{Q})
$$


Let $t_{a}: A \rightarrow A$ denote the translation map defined by $x \mapsto x+a, x \in A$, and $i_{a}: Y_{a} \rightarrow A$ the inclusion map of the translated subvariety $Y_{a}$. Since $t_{a}^{*}$ acts trivially on $H^{k}(A, \mathbf{Q}),(2.3)$ implies that

$$
\pi^{*}(\gamma) \in \operatorname{ker} i_{a}^{*}: H^{k}(A, \mathbf{Q}) \rightarrow H^{k}\left(A-Y_{a}, \mathbf{Q}\right)
$$

holds for any $a \in A$. Now take a point $a \in A$ such that codimension of $B \cap Y_{a}$ in $B$ is equal to codimension of $Y$ in $A$. Then the commutative diagram

$$
\begin{aligned}
& H^{k}(A, \mathbf{Q}) \stackrel{i_{a}^{*}}{\longrightarrow} H^{k}\left(A-Y_{a}, \mathbf{Q}\right) \\
& \quad \iota^{*} \downarrow \\
& H^{k}(B, \mathbf{Q}) \underset{\left.i_{a}\right|_{B} ^{*}}{\longrightarrow} H^{k}\left(B-B \cap Y_{a}, \mathbf{Q}\right)
\end{aligned}
$$

implies that

$$
\gamma=\left.\iota^{*} \pi^{*}(\gamma) \in \operatorname{ker} i_{a}\right|_{B} ^{*}: H^{k}(B, \mathbf{Q}) \rightarrow H^{k}\left(B-B \cap Y_{a}, \mathbf{Q}\right) .
$$

Thus Lemma 2.1 is proved.

By a similar argument, we obtain the following.

Lemma 2.2. For any abelian variety $A$, let $\gamma \in F_{h}^{p} H^{k}(A, \mathbf{Q})$. Suppose that $\pi_{1}^{*}(\gamma) \in F_{a}^{p} H^{k}\left(A^{m}, \mathbf{Q}\right)$ for some $m \geq 1$, where $\pi_{1}: A^{m} \rightarrow A$ denotes the projection to the first factor. Then $\gamma \in F_{a}^{p} H^{k}(A, \mathbf{Q})$.

Furthermore, Lieberman's theorem enables one to show the following.

Lemma 2.3. Let $A$ be an abelian variety of dimension $n$. Then for any $p$ and $k \leq n, \operatorname{GHC}(p, k, A)$ holds if and only if $\operatorname{GHC}(n-k+p, 2 n-k, A)$.

Proof of Lemma 2.3. Let $\varphi_{H}: H^{k}(A, \mathbf{Q}) \rightarrow H^{k+2}(A, \mathbf{Q})$ denote the map defined by taking the intersection with the class of a hyperplane section $H$. Then by the strong Lefschetz theorem its iteration $L_{H}=\varphi_{H}^{n-k}$ gives an isomorphism $L_{H}: H^{k}(A, \mathbf{Q}) \stackrel{\cong}{\cong} H^{2 n-k}(A, \mathbf{Q})$. Moreover by Lieberman's theorem the inverse map $\Lambda_{H}$ of $L_{H}$ is induced by an algebraic correspondence. It follows that it restricts to an isomorphism $L_{H}: F_{a}^{p} H^{k}(A, \mathbf{Q}) \stackrel{\cong}{\leftrightarrows} F_{a}^{p} H^{2 n-k}(A, \mathbf{Q})$ (see $[5,15.34(4)])$. Since $L_{H}$ and $\Lambda_{H}$ are a morphism of type $(n-k, n-k)$ of Hodge structures, Lemma 2.3 is proved.

\section{$\S 3 . \quad$ Varchenko and Hodge Matrices}

In [3] a matrix, called the additive version of Varchenko matrix (AV-matrix for short), is associated to any hyperplane arrangement $\boldsymbol{A}$ in $\mathbf{R}^{n}$. In this section, we recall the definition and some of its fundamental properties. Thereafter 
we introduce a matrix, called Hodge matrix, the kernel of which will play a decisive role in the investigation of the structure of the ring of Hodge cycles on a certain family of abelian varieties of CM-type.

Let $\boldsymbol{A}=\left\{H_{1}, \ldots, H_{k}\right\}$ be a hyperplane arrangement in $\mathbf{R}^{n}$, and let $R(\boldsymbol{A})=\left\{R_{1}, \ldots, R_{m}\right\}$ denote the set of regions of the complement of the union of $\boldsymbol{A}$. For regions $S, T \in R(\boldsymbol{A})$ the number of hyperplanes in $\boldsymbol{A}$ which separate $S$ and $T$ is denoted by $d(S, T)$. In [8], Varchenko defines a matrix $B=B(\boldsymbol{A})$ with rows and columns indexed by the regions in $R(\boldsymbol{A})$ by the rule that the $(S, T)$-th entry in $B$ is $q^{d(S, T)}$. Inspired by this matrix, we introduced in $[3]$ the matrix

$$
D=D(\boldsymbol{A})=(d(S, T))_{(S, T) \in R(\boldsymbol{A}) \times R(\boldsymbol{A})},
$$

and called it the additive version of Varchenko matrix (abbreviated as $A V$ matrix), the rows and columns being ordered according to the given numbering of $R(\boldsymbol{A})$. Let $V(\boldsymbol{A})=\left\{\sum_{R \in R(\boldsymbol{A})} a_{R} R ; a_{R} \in \mathbf{Q}\right\}$ be the $\mathbf{Q}$-vector space consisting of the formal $\mathbf{Q}$-linear combinations of the elements in $R(A)$. Let us recall the fundamental properties of $A V$-matrices, which are proved in [3]:

Proposition 3.1. $\quad$ (see [3, Proposition 2.1]). For any hyperplane $H \in$ $\boldsymbol{A}$, let $h_{H} \in V(\boldsymbol{A})$ denote the vector whose $R_{j}$-th entry $\left(h_{H}\right)_{R_{j}}$ is defined by the rule

$$
\left(h_{H}\right)_{R_{j}}=\left\{\begin{aligned}
1, & \text { if } H \text { does not separate } R_{j} \text { and } R_{1}, \\
-1, & \text { otherwise. }
\end{aligned}\right.
$$

Let Row $-\operatorname{sp}(D(\boldsymbol{A}))$ denote the subspace of $V(\boldsymbol{A})$ generated by the row vectors of $D(\boldsymbol{A})$. Then we have

$$
\text { Row }-\operatorname{sp}(D(\boldsymbol{A}))=\left\langle h_{H} ; H \in \boldsymbol{A}, \mathbf{1}\right\rangle_{\mathbf{Q}},
$$

where $\mathbf{1}=\sum_{R \in R(\boldsymbol{A})} R \in V(\boldsymbol{A})$, and

$$
\operatorname{dim} \text { Row }-\operatorname{sp}(D(\boldsymbol{A}))=\#(\boldsymbol{A})+1 .
$$

Remark. In [3, Proposition 2.1], we made an assumption that

$(O)$ : there exists a pair $(R, S) \in R(\boldsymbol{A}) \times R(\boldsymbol{A})$ such that $d(R, S)=k(=\# \boldsymbol{A})$,

for the validity of the proposition. After the paper was published, however, Prof. Vojta kindly informed to the author that this assumption holds for any 
hyperplane arrangement. His argument goes like this. Given a hyperplane arrangement $\boldsymbol{A}$ in $\mathbf{R}^{n}$, let $\ell$ be any line which intersects every member of $\boldsymbol{A}$. (There exists such a line as an element of a finite intersection of nonempty Zariski dense open subset of a suitable Grassmann variety.) Then the complement $\ell-\ell \cap\left(\bigcup_{H \in A} H\right)$ has exactly two unbounded connected components $\ell^{\prime}, \ell^{\prime \prime}$, and there exists a region $R^{\prime} \in R(\boldsymbol{A})$ (resp. $R^{\prime \prime} \in R(\boldsymbol{A})$ ) such that $\ell^{\prime} \subset R^{\prime}$ (resp. $\left.\ell^{\prime \prime} \subset R^{\prime \prime}\right)$. Then we have $d\left(R^{\prime}, R^{\prime \prime}\right)=k$, since $R^{\prime}$ and $R^{\prime \prime}$ are separated by every hyperplane in $\boldsymbol{A}$ by their choice.

Let $H(\boldsymbol{A})$ denote the $k$ by $m$ matrix consisting of $k$ row vectors $h_{H_{1}}, \ldots$, $h_{H_{k}}$, and let $\operatorname{Hsp}(\boldsymbol{A})$ denote its row space. We call $H(\boldsymbol{A})$ Hodge matrix associated to the hyperplane arrangement $\boldsymbol{A}$. It follows from Proposition 3.1 that

$$
\operatorname{rank} H(\boldsymbol{A})=\operatorname{dim} \mathbf{H s p}(\boldsymbol{A})=k .
$$

For later use we introduce some more notion. A vector $v=\sum_{T \in R(\boldsymbol{A})} a_{T} T$ $\in V(\boldsymbol{A})$ is said to be integral (resp. nonnegative) if $a_{T} \in \mathbf{Z}, T \in R(\boldsymbol{A})$ (resp. $\left.a_{T} \geq 0, T \in R(\boldsymbol{A})\right)$. Furthermore it is said to be $(0,1)$-vector if $a_{T} \in\{0,1\}, T \in$ $R(\boldsymbol{A})$. The sum $\sum_{T \in R(\boldsymbol{A})} a_{T}$ of the coefficients is called its degree and the half sum $\frac{1}{2} \sum_{T \in R(\boldsymbol{A})}\left|a_{T}\right|$ is called its height.

Remark. As will be seen later, when we can associate an abelian variety $A$ to a hyperplane arrangement, a nonnegative integral vector in the kernel of the Hodge matrix $H(\boldsymbol{A})$ corresponds to a Hodge cycle on some self-product $A^{n}$ of codimension equal to its height. This is the main reason why we call $H(\boldsymbol{A})$ Hodge matrix.

\section{$\S 4$. Generalities on Abelian Varieties of CM-type}

In this section, we recall some general facts about abelian varieties of CMtype and their Hodge rings.

Let $K$ be a Galois CM-extension of $\mathbf{Q}$ with $\operatorname{Gal}(K / \mathbf{Q}) \cong G$. Let $\rho \in G$ denote the complex conjugation.

(4.A) The category of CM Q-algebras (= products of CM-fields) split by $K$ is anti-equivalent to the category of finite right $G$-sets in which $\rho$ has no fixed points by the correspondences,

$$
\begin{aligned}
& F \mapsto \operatorname{Hom}_{\mathbf{Q}-\text { algebra }}(F, K) \text { for } F \text { a CM Q-algebra, } \\
& S \mapsto \operatorname{Hom}_{G}(S, K) \text { for } S \text { a finite right } G \text {-set. }
\end{aligned}
$$


(4.B) A finite right $G$-set $S$, plus the data of $S_{1} \subset S$ with $S$ the disjoint sum of $S_{1}$ and $S_{1} \rho$, give an abelian variety $A$ of CM type (up to isogeny), on which the CM-algebra $F$ corresponding to $S$ acts by endomorphisms: the rational lattice is $F \cong \operatorname{Hom}_{G}(S, K)$ and $A$ is $\mathbf{C}^{S_{1}} /\{$ a lattice in $F\}$.

Remark. For any set $X$ and integer $n \geq 1$, we denote by $X_{(n)}$ the disjoint union of $n$ copies of $X$. We will sometimes identify $X_{(n)}$ with $X \times[1, n]$, where $[1, n]=\{1,2, \ldots, n\}$. In this notation, if an abelian variety $A$ corresponds to $S_{1} \subset S$ as in (4.B), then the self-product $A^{n}, n \geq 1$, corresponds to the disjoint union $\left(S_{1}\right)_{(n)} \subset S_{(n)}$ endowed with natural right $G$-set structure.

We call such an abelian variety as in (4.B) an abelian variety split by K. The Hodge ring (= the ring of Hodge cycles) of $A$ is described as follows (see [2], [3] for detail):

(4.C) The first cohomology group $H^{1}(A, \mathbf{C})$ can be identified with $\mathbf{C}^{S}$, and $S_{1}$ defines a one-parameter subgroup $T$ of $G l\left(\mathbf{C}^{S}\right)$. The Hodge group $H g(A)$ is given by $T$ and its conjugates. As a consequence, the complexification of the Hodge $\operatorname{ring}\left(\subset \Lambda \mathbf{C}^{S}\right)$ admits as basis the set of basis vectors of $\Lambda \mathbf{C}^{S}$ corresponding to subsets $P$ of $S$ with the property that

$$
\#\left(P \cap S_{1} g\right)=(\# P) / 2 \quad \text { for any } \quad g \in G .
$$

More generally if $W \subset H^{k}(A, \mathbf{Q})$ is a rational sub-Hodge structure of level $\ell$, then $W_{\mathbf{C}} \subset \Lambda^{k} \mathbf{C}^{S}$ admits as basis the set of basis vectors of $\Lambda^{k} \mathbf{C}^{S}$ corresponding to subsets $Q \subset S$ with the property that

$$
\max _{g \in G}\left\{\left|\#\left(Q \cap S_{1} g\right)-\#\left(Q \cap S_{1} \rho g\right)\right|\right\}=\ell .
$$

By abuse of language, such a subset is said to be of level $\ell$. For later use we prove some general lemmas. We identify $S_{(n)}$ with $S \times[1, n]$ and denote by $p: S_{(n)} \rightarrow S$ the projection onto the first factor. Let $P \subset S_{(n)}$ be a subset with $\#(P)=k$. Then we denote by $[P] \in \Lambda^{k} \mathbf{C}^{S_{(n)}} \cong H^{k}\left(A^{n}, \mathbf{C}\right)$ the corresponding basis element.

Remark. For definiteness, we fix a total order $\leq$ on $S=\left\{s_{i}, s_{i} \rho ; 1 \leq\right.$ $i \leq \operatorname{dim} A\}$ with $s_{i} \leq s_{j}(i \leq j)$ and $s_{i}<s_{j} \rho$, so that $\left[s_{1}\right] \wedge \cdots \wedge\left[s_{\operatorname{dim} A}\right] \wedge$ $\left[s_{1} \rho\right] \wedge \cdots \wedge\left[s_{\operatorname{dim} A} \rho\right]$ gives rise to a volume element on $A$. This total order is extended naturally an order on $S_{(n)}=S \times[1, n]$, and determines the class $[P]$ unambiguously.

We put $X_{P}=\sum_{t \in P} t \in \mathbf{Z}^{S_{(n)}}$ and call it the characteristic vector of $P$. Furthermore let $\mathbf{d}(P)=\sum_{s \in S} \#\left(p^{-1}(s) \cap P\right) \cdot s \in \mathbf{Z}^{S}$ and call it the distribution 
vector of $P$. We set $\mathbf{d}(P)_{s}=\#\left(p^{-1}(s) \cap P\right)$. A subset $P$ is said to be divisorial if $[P]$ is a divisor class, and primitive if $P$ contains no divisorial subsets. It is easy to see that $P$ is primitive if and only if $d(P)_{s} \cdot d(P)_{s \rho}=0$ for any $s \in S_{1}$. Two subsets $P, Q \subset S_{(n)}$ are said to be distribution-equivalent (d-equivalent for

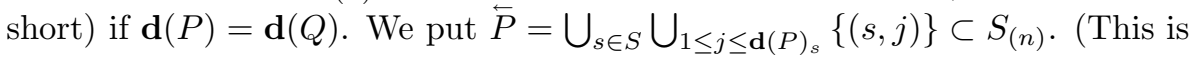
obtained from $P$ by packing it to the left.) Note that $P$ and $\overleftarrow{P}$ are $d$-equivalent, and if $P, Q \subset S_{(n)}$ are $d$-equivalent, then $\overleftarrow{P}=\overleftarrow{Q}$. The following lemmas will be used later when we show the algebraicity of a cohomology class by cutting by divisor classes.

Lemma 4.1. Let $D_{i} \subset S_{(n)}, 1 \leq i \leq m$, be divisorial subsets such that $D_{i} \cap D_{j}=\phi, i \neq j$. Then there exist divisorial subsets $D_{j}^{\prime}, 1 \leq j \leq n \operatorname{dim} A-m$, and $\varepsilon \in\{ \pm 1\}$ such that

$$
H=\varepsilon\left[D_{1}\right]+\sum_{2 \leq i \leq m}\left[D_{i}\right]+\sum_{1 \leq j \leq n \operatorname{dim} A-m}\left[D_{i}^{\prime}\right]
$$

is an ample class.

Proof of Lemma 4.1. Since $S_{(n)}-\coprod_{1<i<m} D_{i}$ is stable under $\rho$, there exists a decomposition $S_{(n)}-\coprod_{1 \leq i \leq m} D_{i}=\coprod_{1 \leq j \leq n \operatorname{dim} A-m} D_{j}^{\prime}$ into divisorial subsets. Then one can check easily that

$$
H^{n \operatorname{dim} A}=\varepsilon(\operatorname{sgn} \sigma)(n \operatorname{dim} A) !\left[S_{(n)}\right],
$$

where $\sigma$ is the permutation on $S_{(n)}$ defined by the partition $S_{(n)}=\coprod_{1<i<m} D_{i}$ $\coprod_{1 \leq j \leq n \operatorname{dim} A-m} D_{j}^{\prime}$. Hence by setting $\varepsilon=\operatorname{sgn} \sigma$, we obtain the ampleness of $H$, since $H^{n \operatorname{dim} A}$ becomes positive.

Therefore we can regard the class $H$ as giving the isomorphism in the strong Lefschetz theorem for $A^{n}$. As an application, we deduce the following crucial result.

Proposition 4.2. Let $P \subset S_{(n)}$ with $\#(P)=k<n \operatorname{dim} A$ be a primitive subset of level $\ell=k-2 p$. Let $D_{i} \subset S_{(n)}, 1 \leq i \leq m$, be mutually disjoint divisorial subsets such that $(P \amalg P \rho) \cap D_{i}=\phi, 1 \leq i \leq m$. Then $[P] \in$ $F_{a}^{p} H^{k}\left(A^{n}, \mathbf{Q}\right)$ if and only if $\left[P \amalg\left(\coprod_{1 \leq i \leq m} D_{i}\right)\right] \in F_{a}^{p+m} H^{k+2 m}\left(A^{n}, \mathbf{Q}\right)$.

Remark. The level of $P \amalg\left(\coprod_{1 \leq i \leq m} D_{i}\right)$ is also equal to $\ell$.

Remark. Our assumption implies $k+m \leq \operatorname{dim} A^{n}$, since $D_{i} \subset S_{(n)}-$ $(P \amalg P \rho)$. 
Proof of Proposition 4.2. The only-if-part is clear. We show the if-part by appealing to Lemma 4.1. Take divisorial subsets $D_{j}^{\prime}, 1 \leq j \leq n \operatorname{dim} A-k-m$, such that

$$
S_{(n)}=(P \coprod P \rho) \coprod\left(\coprod_{1 \leq i \leq m} D_{i}\right) \coprod\left(\coprod_{1 \leq j \leq n \operatorname{dim} A-k-m} D_{j}^{\prime}\right) .
$$

Pick up an element $p \in P$ and put

$$
H=\varepsilon[\{p, p \rho\}]+\sum_{q \in P-\{p\}}[\{q, q \rho\}]+\sum_{1 \leq i \leq m}\left[D_{i}\right]+\sum_{1 \leq j \leq n \operatorname{dim} A-k-m}\left[D_{i}^{\prime}\right]
$$

as in Lemma 4.1. Then it follows from the lemma that $H$ is ample for an appropriate choice of the sign $\varepsilon$. Note that

$$
\begin{aligned}
L_{H}([P]) & =\text { const. }\left[S_{(n)}-P \rho\right] \\
& =\text { const. }\left[P \coprod\left(\coprod_{1 \leq i \leq m} D_{i}\right)\right] \wedge\left[\coprod_{1 \leq j \leq n \operatorname{dim} A-k-m} D_{i}\right] .
\end{aligned}
$$

Hence if $\left[P \amalg\left(\coprod_{1 \leq i \leq m} D_{i}\right)\right] \in F_{a}^{p+m} H^{k+2 m}\left(A^{n}, \mathbf{Q}\right)$, then $L_{H}([P]) \in$ $F_{a}^{\operatorname{dim}} A^{n}-k+p H^{2 \operatorname{dim} A^{n}-k}\left(A^{n}, \mathbf{Q}\right)$, which implies by Lemma 2.3 that $[P] \in$ $F_{a}^{p} H^{k}\left(A^{n}, \mathbf{Q}\right)$. This completes the proof of the proposition.

Next we show the following.

Proposition 4.3. If two primitive subsets $P, Q \subset S_{(n)}$, giving rise to Hodge cycles $[P],[Q]$ on $A^{n}$, are d-equivalent, then $[P]$ is algebraic if and only if $[Q]$ is algebraic.

Proof of Proposition 4.3. Assume that $[P]$ is algebraic. We prove the algebraicity of $[Q]$ by constructing an algebraic correspondence $z$ in $A^{n} \times A^{n}$ such that $z_{*}([P])=[Q]$. Let $\iota_{1}\left(\right.$ resp. $\left.\iota_{2}\right): S_{(n)} \rightarrow S_{(n)} \amalg S_{(n)}$ denote the inclusion map onto the first (resp. second) summand. Let $Z=\iota_{1}\left(S_{(n)}-\right.$ $P) \amalg \iota_{2}(Q)$. Since $P$ and $Q$ are assumed to be $d$-equivalent, we see that

$$
d(Z)_{s}=d\left(S_{(n)}-P\right)_{s}+d(Q)_{s}=d\left(S_{(n)}\right)_{s}-d(P)_{s}+d(Q)_{s}=d\left(S_{(n)}\right)_{s}=n,
$$

hence $d(Z)_{s}$ is constant on $s \in G$, in particular $z=[Z] \in H^{2} \operatorname{dim} A^{n}\left(A^{n} \times A^{n}, \mathbf{Q}\right)$ is an intersection of divisor classes. Let $p_{1}$ (resp. $\left.p_{2}\right)$ denote the projection of 
$A^{n} \times A^{n}$ onto the first (resp. second) factor. Then

$$
\begin{aligned}
z_{*}([P]) & =p_{2 *}\left(p_{1}^{*}[P] . z\right) \\
& =p_{2 *}\left(\left[\iota_{1}(P) \coprod \iota_{1}\left(S_{(n)}-P\right) \coprod \iota_{2}(Q)\right]\right) \\
& =p_{2 *}\left(\left[\iota_{1}\left(S_{(n)}\right) \coprod \iota_{2}(Q)\right]\right) \\
& =[Q] .
\end{aligned}
$$

Therefore the algebraicity of $[P]$ is equivalent to that of $[Q]$. This completes the proof of Proposition 4.3.

Next we recall the definition of $N$-dominatedness and $h$-degeneracy. An abelian variety $A$ of CM-type is said to be $N$-dominated if

for every $n \geq 1$ the Hodge ring $H\left(A^{n}\right)_{\mathbf{C}}$ of $A^{n}$ is

spanned by the Hodge classes $[P], P \subset S_{(n)}$ with

$$
\#(P) \leq 2 N \text {. }
$$

Remark. In [3, Definition 4.5], the notion of $N$-dominatedness is defined only for simple abelian varieties. For nonsimple abelian varieties, we define the notion in exactly the same way. Then one can check easily that when $A$ is of CM-type, it is $N$-dominated if and only if the condition (4.3) holds.

Furthermore an abelian variety $A$ of CM-type is said to be $h$-degenerate if

$$
\begin{aligned}
& \text { for every } n \geq 1 \text { the Hodge ring } H\left(A^{n}\right)_{\mathbf{C}} \text { of } A^{n} \text { is } \\
& \text { spanned by the Hodge classes }[P], P \subset S_{(n)} \text { with } \\
& d(P)_{s} \leq h, s \in S .
\end{aligned}
$$

This notion plays an important role when we try to prove the Hodge Conjecture as follows.

Proposition 4.4. Let $A$ be an abelian variety of CM-type. Suppose that $A$ is $h$-degenerate and the Hodge conjecture holds for any $A^{k}, k \leq h$. Then it holds also for all self-products $A^{n}, n \geq 1$.

Proof of Proposition 4.4. By the definition (4.4), we are reduced to showing that for any $n$, every Hodge classes $[P], P \subset S_{(n)}$ with $d(P)_{s} \leq h, s \in S$, is algebraic. Note that $P$ is $d$-equivalent to $\overleftarrow{P}$ by definition, and the algebraicity of $[\overleftarrow{P}]$, which lives on $A^{h}$, is assured by the assumption. Hence $[P]$ is algebraic by Proposition 4.3 . 
Corollary 4.4.1. Let $A$ be a 1-degenerate abelian variety of CM-type. Suppose that the Hodge conjecture holds for A. Then the whole Hodge conjecture holds for all self-products $A^{n}, n \geq 1$.

\section{§5. Abelian Varieties Associated to CM-arrangements}

In this section, we introduce the notion of CM-arrangement and show that one can associate an abelian variety of CM-type to an arbitrary CMarrangement.

A hyperplane arrangement $\boldsymbol{A}$ in $\mathbf{R}^{n}$ is said to be central when any hyperplane of $\boldsymbol{A}$ contains the origin of $\mathbf{R}^{n}$.

Definition 5.1. A central hyperplane arrangement $\boldsymbol{A}=\left\{H_{1}, \ldots, H_{k}\right\}$ in $\mathbf{R}^{n}$ is said to be a transitive arrangement if there exists a finite group $G \subset$ $G L_{n}(\mathbf{R})$ which acts on the set $\boldsymbol{A}$ transitively. When we want to specify the group $G$, we say $\boldsymbol{A}$ is a $G$-arrangement. The group $G$ is called the structure group of $\boldsymbol{A}$.

In order to associate an abelian variety of CM-type to a $G$-arrangement, we assume the following:

$$
\begin{aligned}
& \text { The group } G \text { is isomorphic to the Galois group of a Galois } \\
& C M \text {-extension } K \text { of } \mathbf{Q} \text {, and the complex conjugation } \\
& \rho \in G \text { acts on } \mathbf{R}^{n} \text { by the multiplication by }-1 \text {. }
\end{aligned}
$$

We call a $G$-arrangement which satisfies the condition (5.1) a CM-arrangement with respect to the pair $(G, K)$.

Remark 5.2. As is illustrated by Example 5.8-5.10, an arrangement $A$ may be acted upon transitively at the same time by different groups, and the isogeny class of the abelian variety attached to $A$ does depend on the pair $(G, K)$. Nevertheless, an important point is that the structure of the Hodge ring of the abelian variety does not depend on the pair (see Corollary 5.5.1).

Now given a CM-arrangement $\boldsymbol{A}$, and for any hyperplane $H \in \boldsymbol{A}$, let $H^{>0}$ denote the connected component of $\mathbf{R}^{n}-H$ which contains the region $R_{1}$, and $H^{<0}$ the other component. Let

$$
\begin{aligned}
& H^{+}=\left\{R \in R(\boldsymbol{A}) ; R \subset H^{>0}\right\}, \\
& H^{-}=\left\{R \in R(\boldsymbol{A}) ; R \subset H^{<0}\right\}\left(=H^{+} \rho\right) .
\end{aligned}
$$


In (4.B), we take the set $R(\boldsymbol{A})$ as $G$-set $S$, and the subset $H_{1}^{+} \subset R(\boldsymbol{A})$ as $S_{1} \subset S$, and denote by $A_{\boldsymbol{A}}(G ; K)$ the abelian variety corresponding to the pair $\left(S, S_{1}\right)=\left(R(\boldsymbol{A}), H_{1}^{+}\right)$. (Note that $\rho$ has no fixed regions in $R(\boldsymbol{A})$ by (5.1).) Since we have assumed that $G$ acts transitively on $\boldsymbol{A}$, the condition (4.1) for a subset $P \subset R(\boldsymbol{A})$ to correspond to a Hodge cycle is equivalent to

$$
\#\left(P \cap H_{i}^{+}\right)=(\# P) / 2 \text { for } 1 \leq i \leq k .
$$

Furthermore this condition is expressed in terms of $\operatorname{Hsp}(\boldsymbol{A})$, introduced in the previous section, as follows. Let $\langle\rangle:, V(\boldsymbol{A}) \times V(\boldsymbol{A}) \rightarrow \mathbf{Q}$ denote the natural inner product on $V(\boldsymbol{A})$ defined by

$$
\left\langle\sum_{R \in R(\boldsymbol{A})} a_{R} R, \sum_{R \in R(\boldsymbol{A})} b_{R} R\right\rangle=\sum_{R \in R(\boldsymbol{A})} a_{R} b_{R} \in \mathbf{Q} .
$$

Then for any $P, Q \subset R(\boldsymbol{A})$, the equality

$$
\#(P \cap Q)=\left\langle X_{P}, X_{Q}\right\rangle
$$

holds. Furthermore note that we can express $h_{H}, H \in \boldsymbol{A}$, in (3.1) as

$$
h_{H}=X_{H^{+}}-X_{H^{-}} .
$$

Proposition 5.5. $\quad A$ subset $P \subset R(\boldsymbol{A})$ gives rise to a Hodge cycle $[P]$ on $A_{\boldsymbol{A}}(G ; K)$ if and only if $X_{P} \in(\boldsymbol{H} \mathbf{s p}(\boldsymbol{A}))^{\perp}$, the orthogonal complement with respect to the inner product $\langle$,$\rangle .$

Proof of Proposition 5.5. This is simply because

$$
\begin{aligned}
& \#\left(P \cap H_{i}^{+}\right)=(\# P) / 2 \\
& \Leftrightarrow \#\left(P \cap H_{i}^{+}\right)=\#\left(P \cap H_{i}^{-}\right) \\
& \Leftrightarrow\left\langle X_{P}, X_{H_{i}^{+}}\right\rangle=\left\langle X_{P}, X_{H_{i}^{-}}\right\rangle \\
& \Leftrightarrow\left\langle X_{P}, h_{H_{i}}\right\rangle=0,
\end{aligned}
$$

and $\operatorname{Hsp}(\boldsymbol{A})$ is, by definition, spanned by $h_{H}, H \in \boldsymbol{A}$.

This implies the following result, which is rather unexpected.

Corollary 5.5.1. The structure of the Hodge ring of $A_{\boldsymbol{A}}(G ; K)$ depends only on $\boldsymbol{A}$, and not on the pair $(G, K)$.

Note that the Hodge group $H g\left(A^{n}\right)$ of the self-product $A^{n}$ of an abelian variety $A$ is isomorphic to the diagonal subgroup of $H g(A)^{n}$ (see [2], [3]). Hence we see that $P \subset R(\boldsymbol{A})_{(n)}$ gives rise to a Hodge cycle on $A_{\boldsymbol{A}}(G ; K)^{n}$ if and only if $\mathbf{d}(P) \in(\mathbf{H s p}(\boldsymbol{A}))^{\perp}$, where $\mathbf{d}(P)$ is the distribution vector defined in Section four. Hence we also have the following. 
Corollary 5.5.2. $\quad$ The structure of the Hodge ring of every self-product $A_{\boldsymbol{A}}(G ; K)^{n}, n \geq 1$, depends only on $\boldsymbol{A}$, and not on the pair $(G, K)$.

We now investigate through (4.A)-(4.C) how the abelian variety $A_{\boldsymbol{A}}(G ; K)$ is decomposed up to isogeny into the product of simple abelian varieties, and give an explicit description of the CM-type of each simple component. Let

$$
R(\boldsymbol{A})=\coprod_{1 \leq \alpha \leq a} O_{\alpha}
$$

be the decomposition of $R(\boldsymbol{A})$ into disjoint orbits under the action of $G$. For any $\alpha \in[1, a]$ and $i \in[1, k]$, let

$$
\begin{aligned}
O_{\alpha} & =\left\{R_{\alpha, 1}, \ldots, R_{\alpha, m_{\alpha}}\right\} \subset R(\boldsymbol{A}) . \\
H_{i, \alpha}^{+} & =H_{i}^{+} \cap O_{\alpha}, \\
H_{i, \alpha}^{-} & =H_{i}^{-} \cap O_{\alpha}\left(=H_{i, \alpha}^{+} \rho\right) .
\end{aligned}
$$

Let $G_{\alpha}$ denote the subgroup of $G$ which fixes the region $R_{\alpha, 1} \in O_{\alpha}$, and let $K_{\alpha}$ denote the subfield of $K$ fixed by $G_{\alpha}$. Note that the field $K_{\alpha}$ is also a CM-field for any $\alpha$ since $\rho \in G$ fixes no regions in $R(A)$ by the assumption (5.1). Furthermore for any $\alpha \in[1, a]$ we have

$$
\left\{H_{i, \alpha}^{+} g ; g \in G\right\}=\left\{H_{i, \alpha}^{+} ; 1 \leq i \leq k\right\},
$$

since $G$ acts on $\boldsymbol{A}$ transitively. It follows from (4.B) that there corresponds an abelian variety of type $\left(K_{\alpha}, H_{1, \alpha}^{+}\right)$, which we denote by $A_{\alpha}\left(G_{\alpha} ; K_{\alpha}\right)$. Moreover we have the following:

Proposition 5.6. For any $\alpha$, the CM-type $H_{1, \alpha}^{+}$for $K_{\alpha}$ is primitive, hence the abelian variety $A_{\alpha}\left(G_{\alpha} ; K_{\alpha}\right)$ is simple.

Proof of Proposition 5.6. First we establish the following general lemma which will be helpful when we need to show the simplicity of given abelian varieties:

Lemma 5.6.1. Let $A$ be an abelian variety of type $\left(E, S_{1}\right)$, and let $L$ be the Galois closure of $E$ in $\mathbf{C}$. Let $G=\operatorname{Gal}(L / \mathbf{Q}), G_{0}=\operatorname{Gal}(L / E)$. Let $\mathbf{Q}[\operatorname{Hom}(E, \mathbf{C})]$ denote the $\mathbf{Q}$-vector space consisting of the formal $\mathbf{Q}$-linear combinations $\sum_{\varphi \in \operatorname{Hom}(E, \mathbf{C})} a_{\varphi} \varphi, a_{\varphi} \in \mathbf{Q}$. Let $\langle$,$\rangle denote the natural inner product$ on $\mathbf{Q}[\operatorname{Hom}(E, \mathbf{C})]$ defined by $\left\langle\sum a_{\varphi} \varphi, \sum b_{\varphi} \varphi\right\rangle=\sum a_{\varphi} b_{\varphi} \in \mathbf{Q}$. Then the abelian variety $A$ is nonsimple if and only if there exists a pair $\{\varphi, \psi\}$ of distinct elements in $\operatorname{Hom}(E, \mathbf{C})$ such that

$$
\left\langle X_{S_{1} g}, \varphi\right\rangle=\left\langle X_{S_{1} g}, \psi\right\rangle \text { holds for any } g \in G \text {. }
$$


(Here $X_{P} \in \mathbf{Q}[\operatorname{Hom}(E, \mathbf{C})]$ denotes for any subset $P \subset \operatorname{Hom}(E, \mathbf{C})$ its characteristic vector.)

Proof of Lemma 5.6.1. Suppose that the condition (5.10) holds. Setting $g=e$, the identity element of $G$, we see that $\varphi$ is not the complex conjugate of $\psi$. Recall that if $S_{1}=\left\{\varphi_{1}, \ldots, \varphi_{n}\right\}$, then the set of pairs

$$
\left\{\varphi_{1}, \varphi_{1} \rho\right\}, \ldots,\left\{\varphi_{n}, \varphi_{n} \rho\right\}
$$

gives rise to an independent set of divisor classes on $A$ (see [2], [3]). Furthermore it follows from [6, Theorem 6$]$ that $A$ is simple if and only if there are no other divisor classes. But if the condition (5.10) holds, the pair $\{\varphi, \psi \rho\}$ defines a divisor class on $A$, since

$$
\begin{aligned}
\#\left(\{\varphi, \psi \rho\} \cap S_{1} g\right) & =\left\langle X_{\{\varphi, \psi \rho\}}, X_{S_{1} g}\right\rangle \\
& =\left\langle X_{\{\varphi\}}, X_{S_{1} g}\right\rangle+\left\langle X_{\{\psi \rho\}}, X_{S_{1} g}\right\rangle \\
& =\left\langle X_{\{\varphi\}}, X_{S_{1} g}\right\rangle+\left\langle X_{\{\psi\}}, X_{S_{1} g \rho}\right\rangle \\
& =\left\langle X_{\{\psi\}}, X_{S_{1} g}+X_{S_{1} g \rho}\right\rangle \\
& =\left\langle X_{\{\psi\}}, X_{\operatorname{Hom}(E, \mathbf{C})}\right\rangle \\
& =1
\end{aligned}
$$

holds for any $g \in G$. But the class defined by $\{\varphi, \psi \rho\}$ is independent of those given by (5.11), hence $A$ is nonsimple. For the converse, take a pair $\{\varphi, \psi\}$ giving rise to a divisor class independent of those corresponding to the pairs in (5.11). Then the same argument as above shows that $\langle S g, \varphi\rangle=\langle S g, \psi \rho\rangle$ holds for any $g \in G$. Thus we complete the proof of Lemma 5.6.1.

Remark 5.6.2. In the statement of the lemma, the condition that " $L$ is the Galois closure of $E$ " is weakened to the one that " $L$ is an arbitrary Galois extension of $\mathbf{Q}$ containing E". For the condition (5.10) holds if and only if $\langle S, \varphi g\rangle=\langle S, \psi g\rangle$ holds for any automorphism $g \in \operatorname{Aut}(\mathbf{C} / \mathbf{Q})$.

Now we go back to the proof of Proposition 5.6. In view of Lemma 5.6.1 and Remark 5.6.2, we are reduced to showing that there exists no pair $\{\varphi, \psi\}$ of distinct elements of $\operatorname{Hom}\left(K_{\alpha}, \mathbf{C}\right)$ such that

$$
\left\langle H_{1, \alpha}^{+} g, \varphi\right\rangle=\left\langle H_{1, \alpha}^{+} g, \psi\right\rangle \text { holds for any } g \in G \text {. }
$$

By the definition of $H_{1, \alpha}^{+}$, we are reduced to showing that there exists no pair of regions $\left\{R_{\alpha, i}, R_{\alpha, j}\right\}$ in $O_{\alpha}$ such that both $R_{\alpha, i}$ and $R_{\alpha, j}$ lie in the same 
connected component of $\mathbf{R}^{n}-H$ for all $H \in \boldsymbol{A}$. But it is clear from the definition of hyperplane arrangement that for any given pair of regions, there exists at least one hyperplane $H$ in $\boldsymbol{A}$ which separates these regions. Thus the proof of Proposition 5.6 is completed.

As a consequence, we have the following:

Proposition 5.7. $\quad$ Notation being as above, the abelian variety $A_{\boldsymbol{A}}(G ; K)$ is decomposed into simple components as $A_{\boldsymbol{A}}(G ; K) \sim \prod_{1 \leq \alpha \leq a} A_{\alpha}\left(G_{\alpha} ; K_{\alpha}\right)$.

We illustrate the above propositions by a few examples. They are concerned with a hyperplane arrangement $\boldsymbol{A}\left(2^{n}\right)$, called the hyperplane arrangement of $(2, \ldots, 2)$-type (or $(2, \ldots, 2)$-arrangement for short), defined as follows. Let $\boldsymbol{A}\left(2^{n}\right)=\left\{H_{1}, \ldots, H_{n}\right\}$ be the hyperplane arrangement in $\mathbf{R}^{n}$ consisting of the coordinate hyperplanes $H_{i}=\left\{\left(x_{1}, \ldots, x_{N}\right) \in \mathbf{R}^{n} ; x_{i}=0\right\}, 1 \leq i \leq n$. Each region of $\boldsymbol{A}\left(2^{n}\right)$ is specified by the sign of the coordinates as follows:

$$
R\left(\boldsymbol{A}\left(2^{n}\right)\right)=\left\{R\left(\varepsilon_{1}, \ldots, \varepsilon_{n}\right) ; \varepsilon_{i} \in\{ \pm 1\}, 1 \leq i \leq n\right\},
$$

where

$$
R\left(\varepsilon_{1}, \ldots, \varepsilon_{n}\right)=\left\{\left(x_{1}, \ldots, x_{n}\right) \in \mathbf{R}^{n} ; \operatorname{sgn}\left(x_{i}\right)=\varepsilon_{i}, 1 \leq i \leq n\right\} .
$$

In particular, we have

$$
\#\left(R\left(\boldsymbol{A}\left(2^{n}\right)\right)\right)=2^{n} .
$$

Example 5.8. Let $C_{3}$, the cyclic group of degree three, act on $\mathbf{R}^{3}$ with coordinate $\left\{x_{1}, x_{2}, x_{3}\right\}$ by the permutation of coordinates, and let $G=\{ \pm i d\} \times$ $C_{3}(\cong \mathbf{Z} / 6 \mathbf{Z}) \subset G L\left(\mathbf{R}^{3}\right)$. Then $\boldsymbol{A}\left(2^{3}\right)$ is visibly a $G$-arrangement. Furthermore, since one can easily construct a Galois CM-field $K$ such that $G a l(K / \mathbf{Q}) \cong G$, the arrangement $\boldsymbol{A}\left(2^{3}\right)$ is a CM-arrangement with respect to the pair $(G, K)$. The orbit decomposition of $R\left(\boldsymbol{A}\left(2^{3}\right)\right)$ under the action of $G$ is given by

$$
R\left(\boldsymbol{A}\left(2^{3}\right)\right)=O_{1} \coprod O_{2},
$$

where

$$
\begin{aligned}
O_{1}=\{R(1,1,1), & R(-1,-1,-1)\}, \\
O_{2}=\{R(1,1,-1), & R(1,-1,1), R(-1,1,1), \\
& R(1,-1,-1), R(-1,1,-1), R(-1,-1,1)\} .
\end{aligned}
$$

Hence we have

$$
\begin{aligned}
& G_{1}=G_{R(1,1,1)}=C_{3}, \\
& G_{2}=G_{R(1,1,-1)}=\{e\} .
\end{aligned}
$$


Therefore $K_{1}=K^{C_{3}}$ is an imaginary quadratic subfield of $K$, and $K_{2}=K$. Let $R_{1}=R(1,1,1)$. Then the set of CM-types $\left\{H_{i, \alpha}^{+} ; i=1,2,3\right\}$ for each $\alpha \in\{1,2\}$ is specified as follows:

$$
\begin{aligned}
& H_{1,1}^{+}=H_{2,1}^{+}=H_{3,1}^{+}=\{R(1,1,1)\}, \\
& H_{1,2}^{+}=\left\{R\left(\varepsilon_{i}\right) ; \varepsilon_{1}=1\right\}=\{R(1,1,-1), R(1,-1,1), R(1,-1,-1)\}, \\
& H_{2,2}^{+}=\left\{R\left(\varepsilon_{i}\right) ; \varepsilon_{2}=1\right\}=\{R(1,1,-1), R(-1,1,1), R(-1,1,-1)\}, \\
& H_{3,2}^{+}=\left\{R\left(\varepsilon_{i}\right) ; \varepsilon_{3}=1\right\}=\{R(1,-1,1), R(-1,1,1), R(-1,-1,1)\} .
\end{aligned}
$$

Therefore we have

$$
A_{\boldsymbol{A}\left(2^{3}\right)}(G ; K) \sim A_{1}\left(G_{1} ; K_{1}\right) \times A_{2}\left(G_{2} ; K_{2}\right),
$$

where $A_{1}\left(G_{1} ; K_{1}\right)$ is an elliptic curve of CM-type and $A_{2}\left(G_{2} ; K_{2}\right)$ is a simple abelian threefold of CM-type. A remarkable fact is that an abelian variety closely related with $A_{A\left(2^{3}\right)}(G ; K)$ arises naturally as the jacobian variety of a hyperelliptic curve. Let $C$ denote the hyperelliptic curve of genus four defined by the equation $y^{2}=x^{9}-1$. The curve $C$ is acted upon by the group $\mu_{9}$ of the ninth roots of unity and we will check shortly that its jacobian variety $J(C)$ is decomposed up to isogeny as

$$
J(C) \sim B_{1} \times B_{2},
$$

where $B_{1}$ is the elliptic curve defined by $y^{2}=x^{3}-1$ and $B_{2}$ is an abelian threefold of CM-type. Since a basis of the space of holomorphic 1-form on $C$ is given by $\omega_{i}=x^{i-1} d x / y(1 \leq i \leq 4)$, the first cohomology space $H^{1}(C, \mathbf{Q}) \cong$ $\left\langle\omega_{i}, \overline{\omega_{i}} ; 1 \leq i \leq 4\right\rangle_{\mathbf{Q}}$ is decomposed as

$$
H^{1}(C, \mathbf{Q}) \cong\left\langle\omega_{3}, \overline{\omega_{3}}\right\rangle_{\mathbf{Q}} \oplus\left\langle\omega_{1}, \omega_{2}, \omega_{4}, \overline{\omega_{1}}, \overline{\omega_{2}}, \overline{\omega_{4}}\right\rangle_{\mathbf{Q}}
$$

as a $\mathbf{Q}$-representation space of $G \cong \operatorname{Gal}\left(\mathbf{Q}\left(\mu_{9}\right) / \mathbf{Q}\right) \cong \mathbf{Z} / 6 \mathbf{Z}$. Hence the jacobian variety is decomposed as in (5.12). The Galois group consists of the six automorphisms $\varphi_{i}: \zeta_{9} \mapsto \zeta_{9}^{i}, i=1,2,4,5,7,8$. The CM-type of $B_{2}$ is given by $T=\left\{\varphi_{1}, \varphi_{2}, \varphi_{4}\right\}$, and its orbit under the action of $G$ consists of

$$
\begin{array}{rlrl}
T & =\left\{\varphi_{1}, \varphi_{2}, \varphi_{4}\right\}, & T \varphi_{2} & =\left\{\varphi_{2}, \varphi_{4}, \varphi_{8}\right\}=\left\{\overline{\varphi_{1}}, \varphi_{2}, \varphi_{4}\right\}, \\
T \varphi_{2}^{2} & =\left\{\varphi_{4}, \varphi_{7}, \varphi_{8}\right\}=\left\{\overline{\varphi_{1}}, \overline{\varphi_{2}}, \varphi_{4}\right\}, & T \varphi_{2}^{3}=T \rho=\left\{\overline{\varphi_{1}}, \overline{\varphi_{2}}, \overline{\varphi_{4}}\right\}, \\
T \varphi_{2}^{4} & =T \varphi_{2} \rho=\left\{\varphi_{1}, \overline{\varphi_{2}}, \overline{\varphi_{4}}\right\}, & T \varphi_{2}^{5} & =T \varphi_{2}^{2} \rho=\left\{\varphi_{1}, \varphi_{2}, \overline{\varphi_{4}}\right\} .
\end{array}
$$


On the other hand, let us give the regions of $A\left(2^{3}\right)$ the following names:

$$
\begin{aligned}
& S_{1}=R(1,1,-1), S_{2}=R(1,-1,-1), S_{4}=R(1,-1,1), \\
& S_{5}=R(-1,1,-1), S_{7}=R(-1,1,1), S_{8}=R(-1,-1,1), \\
& S_{3}=R(1,1,1), S_{6}=R(-1,-1,-1) .
\end{aligned}
$$

Then one can easily check that the correspondence $\varphi_{i} \mapsto S_{i}, 1 \leq i \leq 8$, defines an isomorphism of $G$-sets between $\operatorname{Hom}\left(\mathbf{Q}\left(\mu_{3}\right), \mathbf{C}\right) \amalg \operatorname{Hom}\left(\mathbf{Q}\left(\mu_{9}\right), \mathbf{C}\right)$ and $R\left(A\left(2^{3}\right)\right)$. Moreover note that the CM-type of $A_{1}\left(G_{1} ; K_{1}\right)$ (resp. $A_{2}\left(G_{2} ; K_{2}\right)$ ) is given by $\left\{S_{3}\right\}$ (resp. $\left\{S_{1}, S_{2}, S_{4}\right\}$ ). Hence we obtain the following:

$$
\begin{aligned}
& \text { If we take } \mathbf{Q}\left(\mu_{9}\right) \text { as the } C M \text {-field } K \text { in the } \\
& \text { condition (5.1), the abelian variety } A_{A\left(2^{3}\right)}(G ; K) \text { is } \\
& \text { isogenous to the jacobian variety } J(C) \text { of the } \\
& \text { hyperelliptic curve } C: y^{2}=x^{9}-1 \text {. }
\end{aligned}
$$

Remark 5.9. The arrangement $\boldsymbol{A}\left(2^{3}\right)$ is also acted upon transitively by the wreath product $\{ \pm 1\} \mathrm{wr} S_{3}$, which is the symmetry group of the cube composed of the eight vertices $\{ \pm 1\}^{3} \subset \mathbf{R}^{3}$. If we use this group as the structure group and take an appropriate CM-field $k$, then the associated abelian variety $A_{\boldsymbol{A}\left(2^{3}\right)}\left(\{ \pm 1\} \mathrm{wr} S_{3} ; k\right)$ is isogenous to the "Mumford's example" given in [6]. Since $A_{\boldsymbol{A}\left(2^{3}\right)}\left(\{ \pm 1\} \mathrm{wr} S_{3} ; k\right)$ is simple and $A_{\boldsymbol{A}\left(2^{3}\right)}(G ; K)$ is not, they are not isogenous. Nevertheless the Hodge rings of any power $A_{\boldsymbol{A}\left(2^{3}\right)}\left(\{ \pm 1\} \mathrm{wr} S_{3} ; k\right)^{m}$ and $A_{\boldsymbol{A}\left(2^{3}\right)}(G ; K)^{m}$ are isomorphic for any $m$ by Corollary 5.5.2.

The following is an example of an abelian variety associated to $\boldsymbol{A}\left(2^{2}\right)$. This is simpler than the previous example at a first glance, but tells us that we should be careful when we compute the isogeny decomposition.

Example 5.10. Let $G=\mathbf{Z} / 2 \mathbf{Z} \times \mathbf{Z} / 2 \mathbf{Z}$ acts on $\mathbf{R}^{2}$ by

$$
(1,0) \mapsto\left(\begin{array}{ll}
0 & 1 \\
1 & 0
\end{array}\right), \quad(0,1) \mapsto\left(\begin{array}{rr}
0 & -1 \\
-1 & 0
\end{array}\right) .
$$

Hence $\boldsymbol{A}\left(2^{2}\right)$ is a $G$-arrangement. Take a CM-extension $K$ of $\mathbf{Q}$ of degree four with $\operatorname{Gal}(K / \mathbf{Q}) \cong \mathbf{Z} / 2 \mathbf{Z} \times \mathbf{Z} / 2 \mathbf{Z}$ such that the complex conjugation $\rho$ corresponds to $(1,1) \in G$. Then $\boldsymbol{A}\left(2^{2}\right)$ is a CM-arrangement with respect to the pair $(G, K)$. For definiteness we take $K=\mathbf{Q}(\sqrt{2}, i)$ and let $G$ act on $K$ by

$$
\begin{aligned}
& (1,0): \sqrt{2} \mapsto-\sqrt{2}, i \mapsto i, \\
& (0,1): \sqrt{2} \mapsto-\sqrt{2}, i \mapsto-i .
\end{aligned}
$$


The orbit decomposition of $R\left(\boldsymbol{A}\left(2^{2}\right)\right)$ is given by

$$
\begin{aligned}
& R\left(\boldsymbol{A}\left(2^{2}\right)\right)=O_{1} \coprod O_{2}, \\
& O_{1}=\{R(1,1), R(-1,-1)\} . \\
& O_{2}=\{R(1,-1), R(-1,1)\} .
\end{aligned}
$$

Hence we have

$$
\begin{aligned}
& G_{1}=G_{R(1,1)}=\{(0,0),(1,0)\}, G_{2}=G_{R(1,-1)}=\{(0,0),(0,1)\}, \\
& K_{1}=K^{G_{1}}=\mathbf{Q}(i), K_{2}=K^{G_{2}}=\mathbf{Q}(\sqrt{-2}),
\end{aligned}
$$

and we obtain the isogeny decomposition

$$
A_{\boldsymbol{A}\left(2^{2}\right)}(G, K) \sim E_{1} \times E_{2},
$$

where $E_{1}$ (resp. $E_{2}$ ) is an elliptic curve with complex multiplication by $\mathbf{Q}(i)$ (resp. $\mathbf{Q}(\sqrt{-2}))$. In particular, the simple components are not isogenous. On the other hand, if we take $G^{\prime}=\mathbf{Z} / 4 \mathbf{Z}$, and let it acts on $\mathbf{R}^{2}$ by

$$
1 \mapsto\left(\begin{array}{rr}
0 & -1 \\
1 & 0
\end{array}\right),
$$

then $\boldsymbol{A}\left(2^{2}\right)$ is $G^{\prime}$-arrangement. Since $R\left(\boldsymbol{A}\left(2^{2}\right)\right)$ has one and only one orbit under this action, $A_{\boldsymbol{A}\left(2^{2}\right)}\left(G^{\prime}, K^{\prime}\right)$, for any cyclic CM-field $K^{\prime}$ of degree four, is a simple abelian surface in this case. In particular, $A_{\boldsymbol{A}\left(2^{2}\right)}\left(G^{\prime}, K^{\prime}\right)$ is not isogenous to $A_{\boldsymbol{A}\left(2^{2}\right)}(G, K)$. The Hodge rings of $A_{\boldsymbol{A}\left(2^{2}\right)}(G, K)^{m}$ and $A_{\boldsymbol{A}\left(2^{2}\right)}\left(G^{\prime}, K^{\prime}\right)^{m}$ are, however, well known to be isomorphic for any $m$.

\section{§6. Abelian Varieties of CM-type and the Hyperplane Arrangement of $(2, \ldots, 2)$-type}

In this section we show that any abelian variety of CM-type is realized up to isogeny as an abelian subvariety of an abelian variety associated to a CM-arrangement of $(2, \ldots, 2)$-type.

Let $K$ be a Galois CM-field of degree $2 n$ and let $G=\operatorname{Gal}(K / \mathbf{Q})$. It follows from [1] that there is an injective homomorphism $\Phi: G \rightarrow\{ \pm 1\}$ wr $S_{n}$. For later use we recall briefly the construction of $\Phi$. Let $\rho \in G$ denote the complex conjugation, and let $S_{1}=\left\{g_{1}, \ldots, g_{n}\right\} \subset G$ be a CM-type of $K$ so that $G=\left\{g_{1}, \ldots, g_{n}, g_{1} \rho, \ldots, g_{n} \rho\right\}$. We may assume that $g_{1}$ is the identity of $G$. We define a map $\Sigma: G \rightarrow\{ \pm 1\}^{n}$ by the rule

$$
\Sigma(g)=\left(\varepsilon_{1}, \ldots, \varepsilon_{n}\right) \in\{ \pm 1\}^{n}, \text { where } \varepsilon_{i}=\left\{\begin{aligned}
1, & \text { if } g \in S_{1} g_{i}^{-1} \\
-1, & \text { if } g \notin S_{1} g_{i}^{-1}
\end{aligned}\right.
$$


for $1 \leq i \leq n$. Hence for any $g \in G$, there exists a unique permutation $\sigma=\Pi(g) \in S_{n}$ such that

$$
g g_{i}=\rho^{\mu\left(\varepsilon_{i}\right)} g_{\sigma^{-1}(i)},
$$

where the map $\mu:\{ \pm 1\} \rightarrow \mathbf{Z} / 2 \mathbf{Z}$ is the homomorphism defined by $\mu(1)=$ $0, \mu(-1)=1$.

Proposition 6.1. Notation being as above, let $\Phi: G \rightarrow\{ \pm 1\} \mathrm{wr} S_{n}$ be the map defined by

$$
\Phi(g)=(\Sigma(g) ; \Pi(g))
$$

for any $g \in G$. Then we have the following:

(i) $\Phi$ is an injective homomorphism,

(ii) the image of $\Phi(G)$ under the natural projection $\{ \pm 1\} \mathrm{wr} S_{n} \rightarrow S_{n}$ is a transitive subgroup of $S_{n}$,

(iii) if $A$ is simple then the map $\Sigma: G \rightarrow\{ \pm 1\}^{n}$ is injective.

Proof of Proposition 6.1. The assertions (i) and (ii) are established in [1]. As for the assertion (iii), suppose that the map $\Sigma$ is not injective. Then there exists a pair $\{\varphi, \psi\}$ of distinct elements in $G$ such that $\Sigma(\varphi)=\Sigma(\psi)$. This means by Lemma 5.6.1 that the abelian variety is not simple. This concludes the proof of Proposition 6.1.

We can consider the wreath product $G_{n}=\{ \pm 1\} \mathrm{wr} S_{n}$ naturally as a subgroup of $G L\left(\mathbf{R}^{n}\right)$ by the rule

$$
\left(x_{1}, \ldots, x_{n}\right)\left(\left(\varepsilon_{1}, \ldots, \varepsilon_{n}\right) ; \sigma\right)=\left(\varepsilon_{1} x_{\sigma^{-1}(1)}, \ldots, \varepsilon_{n} x_{\sigma^{-1}(n)}\right) .
$$

Then the homomorphism $\Phi$ provides $\mathbf{R}^{n}$ with the structure of a right $G$-module. Note that the set of hyperplanes $\boldsymbol{A}\left(2^{n}\right)$ is stable under this action of $G_{n}$ on $\mathbf{R}^{n}$, and that, by Proposition 6.1 (ii), $G$ acts transitively on $\boldsymbol{A}\left(2^{n}\right)$. Hence $\boldsymbol{A}\left(2^{n}\right)$ is a CM-arrangement with respect to $(G, K)$ in the sense of Section five. By the definition of the map $\Phi$, we have

$$
\Sigma\left(g_{1}\right)=(1, \ldots, 1) \in\{ \pm 1\}^{n} .
$$

If $A$ is simple, then by Proposition 6.1 (iii), we can identify the set of embeddings $\operatorname{Hom}(K, \mathbf{C})=G$ with the orbit $O_{1}$ of $R_{1}=R(1, \ldots, 1)=R\left(\Sigma\left(g_{1}\right)\right) \in$ $R\left(\boldsymbol{A}\left(2^{n}\right)\right)$ under the action of $G$, hence in particular we have $G_{1}=G_{R_{1}}=\{e\}$. Therefore $K_{1}=K^{G_{1}}=K$. Furthermore it follows from (6.1) with $i=1$ that the CM-type $S_{1}$ is mapped through $\Sigma$ onto $H_{1,1}^{+}=H_{1}^{+} \cap O_{1}$. Hence we obtain the following: 
Proposition 6.2. Any simple abelian variety of CM-type with Galois $C M$-field $K$ such that $\operatorname{Gal}(K / \mathbf{Q}) \cong G$ is realized as a simple component $A_{\alpha}(G ; K)$ of $A_{\boldsymbol{A}\left(2^{n}\right)}(G ; K)$.

In order to deal with the case when the CM-type is not primitive, we need some extra consideration. Let $E \subset K$ be a CM-subfield and $H$ be the corresponding subgroup of $G=\operatorname{Gal}(K / \mathbf{Q})$. Let $R \subset \operatorname{Hom}(E, \mathbf{C})$ be a CM-type of $E$ and $\bar{R}=\pi^{-1}(R) \subset G$ where $\pi: G \rightarrow H \backslash G$ denotes the natural projection. The following proposition shows how the abelian varieties of type $(E, R)$ and of type $(K, \bar{R})$ are related:

Proposition 6.3. $\quad$ Notation being as above, let $A$ be an abelian variety of type $(E, R)$ and $\bar{A}$ an abelian variety of type $(K, \bar{R})$. Then $\bar{A}$ is isogenous to the power $A^{m}$, where $m=[K: E](=\# H)$.

Proof of Proposition 6.3. See Lang [4, Theorem 3.1, 3.4].

The following proposition shows that the converse holds too.

Proposition 6.4. Let $A$ be an abelian variety of type $(K, S)$ with $K$ galois CM-field, and let $G=\operatorname{Gal}(K / \mathbf{Q})$. Let $H=\{g \in G: S g=S\}$ be the stabilizer of $S$, and $\pi(S)=R \subset H \backslash G$ be the image of $S$ under the natural projection. Let $B$ be an abelian variety of type $\left(K^{H}, R\right)$. Then $A$ is isogenous to $B^{\#(H)}$. Moreover $B$ is simple.

Proof of Proposition 6.4. The first part is merely a rephrase of Proposition 6.3. The assertion about the simplicity of $B$ is assured by the criterion given in [7].

These two propositions imply that any abelian variety split by $K$ is isogenous to the product of a number of simple components of abelian varieties of type $\left(K, S_{1}\right)$ with suitable $S_{1}$ 's. Hence by Proposition 6.2 we have the following.

Proposition 6.5. Any abelian variety split by $K$ is realized up to isogeny as an abelian subvariety of a certain self-product $A_{\boldsymbol{A}\left(2^{n}\right)}(G ; K)^{m}$ for suitable $m \geq 1$.

\section{$\S 7$. Kernel of the Hodge Matrix for $\boldsymbol{A}\left(2^{n}\right)$}

In this section, we investigate the structure of the kernel of the Hodge matrix associated to the hyperplane arrangement $\boldsymbol{A}\left(2^{n}\right)$. As a result we will see that for any pair $(G, K)$, the abelian variety $A_{\boldsymbol{A}\left(2^{n}\right)}(G ; K)$ is 2-dominated in the sense of (4.3) and 1-degenerate in the sense of (4.4). 
Since the structure of the Hodge ring of $A_{\boldsymbol{A}\left(2^{n}\right)}(G ; K)^{m}, m \geq 1$, does not depend on the pair $(G, K)$ by Corollary 5.5 .2 , we can take any $(G, K)$ for the investigation of the Hodge ring under the assumption that $A\left(2^{n}\right)$ is a CM-arrangement with respect to $(G, K)$. Accordingly we set $G=\{ \pm 1\} \mathrm{wr} S_{n}$. Note that it has a commutative subgroup $\left\{\left(\left(\varepsilon_{1}, \ldots, \varepsilon_{n}\right) ; e\right) ; \varepsilon_{i} \in\{ \pm 1\}, i \in\right.$ $[1, n]\}$, which is isomorphic to $B_{n}=(\mathbf{Z} / 2 \mathbf{Z})^{n}$. Let $K$ be a Galois CM-field with $\operatorname{Gal}(K / \mathbf{Q}) \cong G$ such that the complex conjugation corresponds to $\mathbf{1}=$ $(1, \ldots, 1) \in B_{n}$. We let $G$ act on $\mathbf{R}^{n}$ as in (6.2). Then $\mathbf{a}=\left(a_{1}, \ldots, a_{n}\right) \in B_{n}$ act on $\mathbf{R}^{n}$ through $\mathbf{a} \mapsto \operatorname{diag}\left((-1)^{a_{1}}, \ldots,(-1)^{a_{n}}\right) \in G L\left(\mathbf{R}^{n}\right)$. Therefore $\boldsymbol{A}\left(2^{n}\right)$ becomes a CM-arrangement with respect to the pair $(G, K)$. From now on we analyze the structure of $V\left(\boldsymbol{A}\left(2^{n}\right)\right)$ as $B_{n}$-module. Since $B_{n}$ is commutative, we need not to discriminate the right and left actions, and we employ the left action. Since the action of $B_{n}$ on the set $R\left(\boldsymbol{A}\left(2^{n}\right)\right)$ of regions is simple and transitive, we can identify $R\left(\boldsymbol{A}\left(2^{n}\right)\right)$ with $B_{n}$ by the rule

$$
\left(a_{i}\right) \in B_{n} \quad \text { corresponds to } R\left((-1)^{a_{1}}, \ldots,(-1)^{a_{n}}\right) .
$$

From now on we call a region of $R\left(A\left(2^{n}\right)\right)$ by the name of the corresponding element of $B_{n}$. Under this identification the function $d(\cdot, \cdot)$ introduced in Section three for an arbitrary arrangement coincides with the so-called Hamming distance. Furthermore the $\mathbf{Q}$-vector space $V\left(\boldsymbol{A}\left(2^{n}\right)\right)$ spanned by $R\left(\boldsymbol{A}\left(2^{n}\right)\right)$ is isomorphic as representation space of $B_{n}$ to the group algebra $\mathbf{Q}\left[B_{n}\right]$. For ease of description we introduce a function $f:\{0,1\}^{n} \rightarrow\left\{0,1, \ldots, 2^{n}-1\right\}$ defined by $f\left(\left(a_{i}\right)\right)=\sum_{1 \leq j \leq n} a_{j} 2^{(n-j)}$, and let $g:\left\{0,1, \ldots, 2^{n}-1\right\} \rightarrow\{0,1\}^{n}$ denote its inverse. (Therefore $g$ gives the 2-adic expansion of an integer.) Identifying the set $\{0,1\}^{n}$ with $(\mathbf{Z} / 2 \mathbf{Z})^{n}$, we number the regions of $\boldsymbol{A}\left(2^{n}\right)$ by the following rule:

The region $\left(a_{i}\right) \in B_{n}$ acquires the number $1+f\left(\left(a_{i}\right)\right)$.

Hence, in particular, the first region $R_{1}$ corresponds to the subset $\left\{\left(x_{i}\right) ; x_{i}>\right.$ $0,1 \leq i \leq n\}$.

Our purpose in this section is to show the following:

Theorem 7.1. The kernel of the Hodge matrix $H\left(\boldsymbol{A}\left(2^{n}\right)\right)$ is spanned by $(0,1)$-vectors of height one and two.

Let us introduce some notation and recall some basic facts about the representation theory of $B_{n}$. 
Definition 7.2. $\quad$ For any $\mathbf{a}=\left(a_{i}\right) \in B_{n}$, let $\chi_{\mathbf{a}} \in \operatorname{Hom}\left(B_{n}, \mathbf{C}^{*}\right)$ denote the character of $B_{n}$ defined by

$$
\chi_{\mathbf{a}}(\sigma)=(-1)^{1 \leq i \leq n} a_{i} \sigma_{i} \quad \text { for } \quad \sigma=\left(\sigma_{i}\right) \in B_{n} .
$$

Let $v_{\mathbf{a}}$ denote the vector in $V\left(\boldsymbol{A}\left(2^{n}\right)\right)\left(=\mathbf{Q}\left[B_{n}\right]\right)$ defined by

$$
v_{\mathbf{a}}=\sum_{\sigma \in B_{n}} \chi_{\mathbf{a}}(\sigma) \sigma .
$$

The element $v_{\mathbf{a}}$ gives a basis element of the one-dimensional vector space

$$
V\left(\chi_{\mathbf{a}}\right)=\left\{v \in V\left(\boldsymbol{A}\left(2^{n}\right)\right) ; \sigma v=\chi_{\mathbf{a}}(\sigma) v \text { for any } \sigma \in B_{n}\right\},
$$

which affords the representation $\chi_{\mathbf{a}}$ of $B_{n}$, so that we have

$$
V\left(\boldsymbol{A}\left(2^{n}\right)\right)=\bigoplus_{\mathbf{a} \in B_{n}} V\left(\chi_{\mathbf{a}}\right) .
$$

We want to determine the structures of Row $-\operatorname{sp}\left(\boldsymbol{A}\left(2^{n}\right)\right)$ and $\operatorname{Hsp}\left(\boldsymbol{A}\left(2^{n}\right)\right)$ as representation spaces of $B_{n}$. First we observe the following:

Proposition 7.3. For any $i$ with $1 \leq i \leq n$, we have

$$
h_{H_{i}}=v_{\mathbf{e}_{i}},
$$

where $\mathbf{e}_{i}=(0, \ldots, 0, \stackrel{i}{1}, 0, \ldots, 0) \in B_{n}$. (See (3.1) for the definition of $h_{H_{i}}$.)

Proof of Proposition 7.3. By the definition, we have

$$
h_{H_{i}}=\sum_{T \in R\left(\boldsymbol{A}\left(2^{n}\right)\right)} a_{T} T
$$

where

$$
a_{T}=\left\{\begin{aligned}
1, & \text { if } H_{i} \text { does not separate } R_{1} \text { and } T \\
-1, & \text { otherwise. }
\end{aligned}\right.
$$

Moreover, $H_{i}$ does not separate $R_{1}$ and $T$ if and only if the $i$-th coordinate of the elements of $T$ is positive. The latter condition is equivalent to the equality $\chi_{\mathbf{e}_{i}}(T)=1$. Hence, by the definition of $v_{\mathbf{a}}$, we have

$$
h_{H_{i}}=v_{\mathbf{e}_{i}},
$$

which concludes the proof of Proposition 7.3.

Combining this proposition with Proposition 3.1, we obtain the following. 
Proposition 7.4. As representation spaces of $B_{n}$, we have

$$
\begin{aligned}
\text { Row }-\operatorname{sp}\left(D\left(\boldsymbol{A}\left(2^{n}\right)\right)\right) & =\bigoplus_{1 \leq i \leq n} V\left(\chi_{\mathbf{e}_{i}}\right) \oplus V\left(\chi_{(0, \ldots, 0)}\right), \\
\operatorname{Hsp}\left(\boldsymbol{A}\left(2^{n}\right)\right) & =\bigoplus_{1 \leq i \leq n} V\left(\chi_{\mathbf{e}_{i}}\right) .
\end{aligned}
$$

Now we investigate the kernel of the Hodge matrix $H\left(\boldsymbol{A}\left(2^{n}\right)\right)$. Let $\rho$ : $B_{n} \rightarrow B_{n}$ denote the map defined by $\rho(\mathbf{a})=\mathbf{1}$.a. (Here and from now on we write the group operation of $B_{n}$ multiplicatively in order to avoid a possible confusion between it and the addition in $\mathbf{Q}\left[B_{n}\right]$.) First we construct $(0,1)$ vectors of height one in the kernel.

Definition 7.5. Let $B_{n}^{0}=\left\{\mathbf{a} \in B_{n} ; a_{1}=0\right\}$. For any $\mathbf{a} \in B_{n}^{0}$, let

$$
d_{\mathbf{a}}=\mathbf{a}+\rho(\mathbf{a}) \in V\left(\boldsymbol{A}\left(2^{n}\right)\right) .
$$

Remark 7.6. By (4.1), these elements give rise to divisor classes on the abelian variety $A_{\boldsymbol{A}\left(2^{n}\right)}(G ; K)$.

Note that the natural inner product $\langle$,$\rangle on V\left(\boldsymbol{A}\left(2^{n}\right)\right)$, defined by

$$
\left\langle\sum_{T \in R\left(\boldsymbol{A}\left(2^{n}\right)\right)} a_{T} T, \sum_{T \in R\left(\boldsymbol{A}\left(2^{n}\right)\right)} b_{T} T\right\rangle=\sum_{T \in R\left(\boldsymbol{A}\left(2^{n}\right)\right)} a_{T} b_{T}(\in \mathbf{Q}),
$$

is $B_{n}$-equivariant, and that the kernel of the Hodge matrix coincides with the orthogonal complement $\mathbf{H} \mathbf{s p}\left(\boldsymbol{A}\left(2^{n}\right)\right)^{\perp}$ of $\mathbf{H s p}\left(\boldsymbol{A}\left(2^{n}\right)\right)$ in $V\left(\boldsymbol{A}\left(2^{n}\right)\right)$ with respect to the inner product. We prove the following:

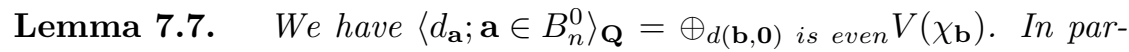
ticular, every $d_{\mathbf{a}}$ belongs to the kernel of the Hodge matrix $H\left(\boldsymbol{A}\left(2^{n}\right)\right)$.

Proof of Lemma 7.7. For any $\mathbf{a}, \mathbf{b} \in B_{n}$, we have

$$
\begin{aligned}
\left\langle\mathbf{a}, v_{\mathbf{b}}\right\rangle & =\chi_{\mathbf{b}}(\mathbf{a}), \\
\left\langle\rho(\mathbf{a}), v_{\mathbf{b}}\right\rangle & =\left\langle\mathbf{1} . \mathbf{a}, v_{\mathbf{b}}\right\rangle=\left\langle\mathbf{a}, \mathbf{1} . v_{\mathbf{b}}\right\rangle=\chi_{\mathbf{b}}(\mathbf{1})\left\langle\mathbf{a}, v_{\mathbf{b}}\right\rangle=(-1)^{d(\mathbf{b}, \mathbf{0})} \chi_{\mathbf{b}}(\mathbf{a}) .
\end{aligned}
$$

Therefore

$$
\begin{aligned}
\left\langle d_{\mathbf{a}}, v_{\mathbf{b}}\right\rangle & =\left\langle\mathbf{a}, v_{\mathbf{b}}\right\rangle+\left\langle\rho(\mathbf{a}), v_{\mathbf{b}}\right\rangle=\chi_{\mathbf{b}}(\mathbf{a})\left(1+(-1)^{d(\mathbf{b}, \mathbf{0})}\right) \\
& = \begin{cases}2 \chi_{\mathbf{b}}(\mathbf{a}), & \text { if } d(\mathbf{b}, \mathbf{0}) \text { is even, } \\
0, & \text { if } d(\mathbf{b}, \mathbf{0}) \text { is odd } .\end{cases}
\end{aligned}
$$


It follows that

$$
d_{\mathbf{a}} \in \bigoplus_{d(\mathbf{b}, \mathbf{0}) \text { is even }} V\left(\chi_{\mathbf{b}}\right)
$$

Since the supports of $d_{\mathbf{a}}$ for $\mathbf{a}=\left(a_{1}, \ldots, a_{n}\right) \in B_{n}^{0}$ are mutually disjoint, they are linearly independent in $V\left(\boldsymbol{A}\left(2^{n}\right)\right)$ and generate a $2^{n-1}$-dimensional subspace. Since the dimension of the space on the right hand side of (7.2) is equal to $2^{n-1}$ too, the first assertion is proved. The second assertion follows from Proposition 7.4 by the orthogonality relations of characters.

Next we construct $(0,1)$-vectors of height two in the kernel of the Hodge matrix $H\left(\boldsymbol{A}\left(2^{n}\right)\right)$.

Definition 7.8. For any pair $(i, j)$ with $1 \leq i<j \leq n$, let

$$
z_{i j}=\mathbf{0}+\rho\left(\mathbf{e}_{i}\right)+\rho\left(\mathbf{e}_{j}\right)+\mathbf{e}_{i j} \in V\left(\boldsymbol{A}\left(2^{n}\right)\right)
$$

where

$$
\mathbf{0}=(0, \ldots, 0), \mathbf{e}_{i j}=(0, \ldots, 0, \stackrel{\mathrm{i}}{1}, 0, \ldots, 0, \stackrel{\mathrm{i}}{1}, 0, \ldots, 0) \in B_{n} .
$$

Lemma 7.9. Every $z_{i j}$ belongs to the kernel of the Hodge matrix $H\left(\boldsymbol{A}\left(2^{n}\right)\right)$.

Proof of Lemma 7.9. An element $v \in V\left(\boldsymbol{A}\left(2^{n}\right)\right)$ belongs to the kernel of $H\left(\boldsymbol{A}\left(2^{n}\right)\right)$ if and only if $\langle v, w\rangle=0$ for any $w \in \mathbf{H} \mathbf{s p}\left(\boldsymbol{A}\left(2^{n}\right)\right)$. Note that

$$
\left\langle\mathbf{e}_{i}, h_{H_{j}}\right\rangle=\left\langle\mathbf{e}_{i}, \mathbf{v}_{\mathbf{e}_{j}}\right\rangle=\chi_{\mathbf{e}_{j}}\left(\mathbf{e}_{i}\right)=\left\{\begin{aligned}
1, & \text { if } i \neq j, \\
-1, & \text { if } i=j .
\end{aligned}\right.
$$

Hence

$$
\begin{aligned}
\left\langle\rho\left(\mathbf{e}_{i}\right), h_{H_{j}}\right\rangle & =\left\langle\mathbf{1} . \mathbf{e}_{i}, h_{H_{j}}\right\rangle=\left\langle\mathbf{e}_{i}, \mathbf{1} \cdot h_{H_{j}}\right\rangle=\left\langle\mathbf{e}_{i}, \mathbf{1} \cdot \mathbf{v}_{\mathbf{e}_{j}}\right\rangle=\chi_{\mathbf{e}_{j}}(\mathbf{1})\left\langle\mathbf{e}_{i}, \mathbf{v}_{\mathbf{e}_{j}}\right\rangle \\
& =\left\{\begin{aligned}
-1, & \text { if } i \neq j, \\
1, & \text { if } i=j .
\end{aligned}\right.
\end{aligned}
$$

On the other hand, we have

$$
\left\langle\mathbf{e}_{i j}, h_{H_{k}}\right\rangle=\left\langle\mathbf{e}_{i j}, \chi_{\mathbf{e}_{k}}\right\rangle=\chi_{\mathbf{e}_{k}}\left(\mathbf{e}_{i j}\right)=\left\{\begin{aligned}
1, & \text { if } k \neq i, j, \\
-1, & \text { if } k=i \text { or } k=j .
\end{aligned}\right.
$$

Therefore, if $k \neq i, j$, then it follows from (7.3) that

$$
\left\langle z_{i j}, h_{H_{k}}\right\rangle=1-1-1+1=0 .
$$


Moreover we have

$$
\begin{gathered}
\left\langle z_{i j}, h_{H_{i}}\right\rangle=1+1-1-1=0, \\
\left\langle z_{i j}, h_{H_{j}}\right\rangle=1-1+1-1=0 .
\end{gathered}
$$

By the equalities (7.4)-(7.6), we see that $z_{i j}$ belongs to the kernel of the Hodge matrix $H\left(\boldsymbol{A}\left(2^{n}\right)\right)$. This concludes the proof of Lemma 7.9.

Since $\operatorname{Hsp}\left(\boldsymbol{A}\left(2^{n}\right)\right)$ is $B_{n}$-stable by Proposition 7.4, we also have the following:

Lemma 7.10. For any $i, j$ and $\sigma \in B_{n}$, the element $\sigma z_{i j}$ belongs to the kernel of the Hodge matrix $H\left(\boldsymbol{A}\left(2^{n}\right)\right)$.

Next we show that every element of $\oplus_{d(\mathbf{b}, \mathbf{0}) \geq 3} V\left(\chi_{\mathbf{b}}\right)$ is a linear combination of $\sigma z_{i j}$. A crucial point is given by the following.

Lemma 7.11. Assume that $n \geq 3$. Then for any $\mathbf{c} \in B_{n-3}$, we have

$$
v_{(111 \mathbf{c})}=\sum_{\sigma \in B_{n-3}} \chi_{\mathbf{c}}(\sigma)((000 \sigma)-(111 \sigma)) z_{12} .
$$

Proof of Lemma 7.11. This is shown by the following computation:

$$
\begin{aligned}
v_{(111 \mathbf{c})=} & \sum_{\tau \in B_{n}} \chi_{(111 \mathbf{c})}(\tau) \tau \\
= & \sum_{\sigma \in B_{n}}\left(\chi_{(111 \mathbf{c})}(000 \sigma) \cdot(000 \sigma)+\chi_{(111 \mathbf{c})}(001 \sigma) \cdot(001 \sigma)\right. \\
& +\chi_{(111 \mathbf{c})}(010 \sigma) \cdot(010 \sigma)+\chi_{(111 \mathbf{c})}(100 \sigma) \cdot(100 \sigma) \\
& +\chi_{(111 \mathbf{c})}(011 \sigma) \cdot(011 \sigma)+\chi_{(111 \mathbf{c})}(101 \sigma) \cdot(101 \sigma) \\
& \left.+\chi_{(111 \mathbf{c})}(110 \sigma) \cdot(110 \sigma)+\chi_{(111 \mathbf{c})}(111 \sigma) \cdot(111 \sigma)\right) \\
= & \sum_{\sigma \in B_{n-3}}\left(\chi_{\mathbf{c}}(\sigma) \cdot(000 \sigma)-\chi_{\mathbf{c}}(\sigma) \cdot(001 \sigma)-\chi_{\mathbf{c}}(\sigma) \cdot(010 \sigma)-\chi_{\mathbf{c}}(\sigma) \cdot(100 \sigma)\right. \\
& \left.+\chi_{\mathbf{c}}(\sigma) \cdot(011 \sigma)+\chi_{\mathbf{c}}(\sigma) \cdot(101 \sigma)+\chi_{\mathbf{c}}(\sigma) \cdot(110 \sigma)-\chi_{\mathbf{c}}(\sigma) \cdot(111 \sigma)\right) \\
= & \sum_{\sigma \in B_{n-3}} \chi_{\mathbf{c}}(\sigma)((000 \sigma)-(001 \sigma)-(010 \sigma)-(100 \sigma) \\
& +(011 \sigma)+(101 \sigma)+(110 \sigma)-(111 \sigma)) \\
= & \sum_{\sigma \in B_{n-3}} \chi_{\mathbf{c}}(\sigma)((000 \sigma)-(111 \sigma)) z_{12} .
\end{aligned}
$$

By using a suitable permutation on $[1, n]$, we can convert this lemma into the following form. 
Lemma 7.12. Suppose $n \geq 3$. Then for any triple $(i, j, k)$ with $1 \leq i<$ $j<k \leq n$ and for any $\mathbf{b} \in B_{n}$ with $b_{i}=b_{j}=b_{k}=1$, the element $v_{\mathbf{b}}$ belongs to $\left\langle\sigma z_{i j} ; \sigma \in B_{n}\right\rangle_{\mathbf{Q}}$. In particular, we have the inclusion

$$
\bigoplus_{d(\mathbf{b}, \mathbf{0}) \geq 3} V\left(\chi_{\mathbf{b}}\right) \subset\left\langle\sigma z_{i j} ; 1 \leq i<j \leq n, \sigma \in B_{n}\right\rangle_{\mathbf{Q}} .
$$

Combining this lemma with Lemma 7.7 and Proposition 7.4, we obtain the following.

Theorem 7.13. $\quad$ The kernel of the Hodge matrix $H\left(\boldsymbol{A}\left(2^{n}\right)\right)$ is spanned by

$$
\sigma d_{\mathbf{a}}, \text { with } \mathbf{a} \in B_{n}^{0}, \sigma \in B_{n},
$$

and

$$
\sigma z_{i j}, \text { with } 1 \leq i<j \leq n, \sigma \in B_{n} .
$$

Thus Theorem 7.13 implies by Proposition 4.9 in [3] the following:

Theorem 7.14. When $n=1,2$, the abelian variety $A_{\boldsymbol{A}\left(2^{n}\right)}(G ; K)$ is 1-dominated, namely, nondegenerate. When $n \geq 3$, the abelian variety $A_{A\left(2^{n}\right)}(G ; K)$ is 1-degenerate and 2-dominated.

By Corollary 4.4.1, this implies the following.

Corollary 7.14.1. If every Hodge cycle of codimension two on $A_{\boldsymbol{A}\left(2^{n}\right)}(G ; K)$ is algebraic, then the whole Hodge conjecture holds true for any self-products $A_{\boldsymbol{A}\left(2^{n}\right)}(G ; K)^{m}, m \geq 1$.

Remark 7.15. The $N$-dominatedness does not imply that every element in the kernel of the Hodge matrix is expressed as a linear combination of integral vectors of height $N$ with nonnegative coefficients. When $n=4$, for example, the element

$$
v=(0,0,0,0)+(0,0,1,1)+(0,1,0,1)+(1,0,0,1)+2(1,1,1,0) \in V\left(\boldsymbol{A}\left(2^{4}\right)\right)
$$

belongs to the kernel of the Hodge matrix $H\left(\boldsymbol{A}\left(2^{4}\right)\right)$, and one can check that $v$ cannot be expressed as a linear combination of the elements in Theorem 7.12 with nonnegative coefficients. But it is expressed simply as

$$
v=z_{24}+z_{34}-z_{23}+d_{0110},
$$

and hence the theory developed in [3] assures that the algebraicity of the Hodge cycle corresponding to $v$ on $A_{\boldsymbol{A}\left(2^{4}\right)}(G ; K)^{2}$ is implied by that of the Hodge cycles corresponding to $z_{24}, z_{34}, z_{23}$. 


\section{§. General Hodge Conjecture for Abelian Varieties of CM-type}

In this section we show that if every Hodge cycle of codimension two on the abelian varieties of $(2, \ldots, 2)$-type is algebraic, then the whole GHC holds for them. As a result we obtain the validity of GHC for arbitrary abelian varieties of CM-type under the same hypothesis.

Notation being as in Section five, we express GHC for the abelian varieties associated to $\boldsymbol{A}\left(2^{n}\right)$ in terms of some linear algebra. In view of Proposition 6.1, we may assume that we are given a pair $(G, K)$ such that

$$
\begin{aligned}
& G \text { is isomorphic to the Galois group of a Galois } \\
& \text { CM-extension field Kover } \mathbf{Q} \text {. } \\
& G \text { is isomorphic to a subgroup of the wreath } \\
& \text { product } G_{n}=\{ \pm 1\} \mathrm{wr}_{n} \text {, and through the embedding } \\
& G_{n} \rightarrow G l\left(\mathbf{R}^{n}\right) \text { in Proposition } 6.1 \text { G acts on } \boldsymbol{A}\left(2^{n}\right) \\
& \text { transitively. }
\end{aligned}
$$

Let $A=A_{\boldsymbol{A}\left(2^{n}\right)}(G ; K)$ and $S=R\left(\boldsymbol{A}\left(2^{n}\right)\right)$. Then applying the generalities in (4.C) to our abelian variety, we obtain the following.

Proposition 8.1. If $W \subset H^{k}(A, \mathbf{Q})$ is a rational sub-Hodge structure of level $\ell$, then $W_{\mathbf{C}} \subset \Lambda^{k} \mathbf{C}^{S}$ admits as basis the set of basis vectors of $\Lambda^{k} \mathbf{C}^{S}$ corresponding to subsets $Q \subset S$ with the property that

$$
\max _{1 \leq i \leq n}\left\{\left|\left\langle X_{Q}, h_{H_{i}}\right\rangle\right|\right\}=\ell .
$$

Remark. For the reason of parity, the condition (8.3) is equivalent to

$$
\begin{aligned}
& \left\langle X_{Q}, h_{H_{i}}\right\rangle \in\{-\ell,-\ell+2, \ldots, \ell-2, \ell\} \text { for any } i, \\
& \text { and }\left|\left\langle X_{Q}, h_{H_{i}}\right\rangle\right|=\ell \text { for some } i .
\end{aligned}
$$

Now we can prove the following theorem which is one of the main results of this paper.

Theorem 8.2. $\quad$ Suppose that the Hodge cycles of codimension two on $A_{\boldsymbol{A}\left(2^{n}\right)}(G ; K)$ is algebraic. Then the whole $G H C$ holds for $A_{\boldsymbol{A}\left(2^{n}\right)}(G ; K)$.

Proof of Theorem 8.2. We prove the theorem in the form of (2.2). Let $W \subset H^{k}(A, \mathbf{Q})$ be a rational sub-Hodge structure with $\ell(W)=\ell$. We have 
to show that $W$ is supported on a Zariski-closed subset of $A$ of codimension $(k-\ell) / 2$.

Case 0) When $\ell=0$ : This case reduces to the usual Hodge Conjecture. Since we have seen in Theorem 7.11 that the abelian variety $A$ is 2 -dominated, the assumption implies the validity of the Hodge Conjecture by [3].

Case 1) When $\ell=1$ : Let $W \subset H^{k}(A, \mathbf{Q})$ be a rational sub-Hodge structure of level one. (Hence $k$ is necessarily odd.) Then by Proposition 8.1, $W_{\mathbf{C}} \subset \Lambda^{k} \mathbf{C}^{S}$ admits as basis the set of basis vectors of $\Lambda^{k} \mathbf{C}^{S}$ corresponding to subsets $Q \subset S$ satisfying (8.3) with $\ell=1$. When $k=1$, there is nothing to prove. We prove the assertion by induction on $k$. Therefore we may assume that $k \geq 3$ and that the assertion is proved for smaller odd integers. In view of this assumption, we may assume that $Q$ is primitive, namely

$$
Q \cap \rho(Q)=\phi,
$$

because if $Q \cap \rho(Q) \neq \phi$, the assertion is reduced to that for smaller $k$. The condition (8.4) says that

$$
\left\langle X_{Q}, h_{H_{i}}\right\rangle \in\{ \pm 1\}
$$

holds for any $i$. This means that there exists a unique $\mathbf{a}=\left(a_{i}\right) \in B_{n}$ such that

$$
\left\langle X_{Q}, h_{H_{i}}\right\rangle=(-1)^{a_{i}}, 1 \leq i \leq n .
$$

Since

$$
\left\langle\mathbf{a}, h_{H_{i}}\right\rangle=\left\langle\mathbf{a}, v_{\mathbf{e}_{i}}\right\rangle=\chi_{\mathbf{e}_{i}}(\mathbf{a})=(-1)^{a_{i}},
$$

it follows from (8.6) and (8.7) that

$$
\left\langle X_{Q}-\mathbf{a}, h_{H_{i}}\right\rangle=0,1 \leq i \leq n,
$$

hence $X_{Q}-\mathbf{a}$ belongs to the kernel of the Hodge matrix $H=H\left(\boldsymbol{A}\left(2^{n}\right)\right)$. Since the abelian variety $A$ is 2-dominated, Proposition 4.9 of [3] implies the existence of a positive integer $n$, a number of four-element subsets $M_{1}, \ldots, M_{p} \subset S_{(n)}$ with $M_{i} \cap \rho\left(M_{i}\right)=\phi$, and elements $s_{1}, \ldots, s_{q} \in\left(S_{1}\right)_{(n)}$ such that

$$
\begin{aligned}
X_{Q}-\mathbf{a}= & \sum_{1 \leq \alpha \leq p^{\prime}} X_{M_{\alpha}}-\sum_{p^{\prime}+1 \leq \beta \leq p} X_{M_{\beta}}+\sum_{1 \leq \gamma \leq q^{\prime}} X_{\left\{s_{\gamma}, \rho s_{\gamma}\right\}} \\
& -\sum_{\substack{q^{\prime}+1 \leq \delta \leq q \\
X_{\left\{s_{\delta}, \rho s_{\delta}\right\}},}} X_{M_{\alpha}=}, 1 \leq \alpha \leq p .
\end{aligned}
$$


It follows that the equality

$$
\begin{aligned}
X_{Q} & +\sum_{p^{\prime}+1 \leq \beta \leq p} X_{M_{\beta}}+\sum_{q^{\prime}+1 \leq \delta \leq q} X_{\left\{s_{\delta}, \rho s_{\delta}\right\}} \\
& =\sum_{1 \leq \alpha \leq p^{\prime}} X_{M_{\alpha}}+\sum_{1 \leq \gamma \leq q^{\prime}} X_{\left\{s_{\gamma}, \rho s_{\gamma}\right\}}+\mathbf{a}
\end{aligned}
$$

holds, and hence adding $\sum_{p^{\prime}+1 \leq \beta \leq p} X_{\rho\left(M_{\beta}\right)}$ to both sides we have

$$
\begin{aligned}
& X_{Q}+\sum_{p^{\prime}+1 \leq \beta \leq p} X_{M_{\beta}}+\left(\sum_{p^{\prime}+1 \leq \beta \leq p} X_{\rho\left(M_{\beta}\right)}\right)+\sum_{q^{\prime}+1 \leq \delta \leq q} X_{\left\{s_{\delta}, \rho s_{\delta}\right\}} \\
& =\sum_{1 \leq \alpha \leq p^{\prime}} X_{M_{\alpha}}+\left(\sum_{p^{\prime}+1 \leq \beta \leq p} X_{\rho\left(M_{\beta}\right)}\right)+\sum_{1 \leq \gamma \leq q^{\prime}} X_{\left\{s_{\gamma}, \rho s_{\gamma}\right\}}+\mathbf{a} .
\end{aligned}
$$

Since $\left[M_{\alpha}\right]$ and $\left[\rho\left(M_{\beta}\right)\right]$ is algebraic by the assumption of the theorem, this equality implies that

$$
\begin{aligned}
& {\left[Q \coprod\left(\coprod_{p^{\prime}+1 \leq \beta \leq p}\left(M_{\beta} \coprod \rho\left(M_{\beta}\right)\right)\right) \coprod\left(\coprod_{q^{\prime}+1 \leq \delta \leq q}\left\{s_{\delta}, \rho s_{\delta}\right\}\right)\right]} \\
& \in F_{a}^{(k-1) / 2+\left(p-p^{\prime}\right)+\left(q-q^{\prime}\right)} H^{k+\left(p-p^{\prime}\right)+\left(q-q^{\prime}\right)}\left(A^{n}, \mathbf{Q}\right) .
\end{aligned}
$$

Since $\left(\coprod_{p^{\prime}+1 \leq \beta \leq p}\left(M_{\beta} \amalg \rho\left(M_{\beta}\right)\right)\right) \amalg\left(\coprod_{q^{\prime}+1 \leq \delta \leq q}\left\{s_{\delta}, \rho s_{\delta}\right\}\right)$ is a disjoint union of divisorial subsets, Proposition 4.2 implies that

$$
[Q] \in F_{a}^{(k-1) / 2} H^{k}\left(A^{n}, \mathbf{Q}\right) .
$$

Thus the theorem is proved in this case.

Case 2) When $\ell \geq 2$ : The idea behind our proof is basically similar to that employed in Case 1). Let $W \subset H^{k}(A, \mathbf{Q})$ be a rational sub-Hodge structure of level $\ell$. Then $W_{\mathbf{C}} \subset \Lambda^{k} \mathbf{C}^{S}$ admits as basis the set of basis vectors of $\Lambda^{k} \mathbf{C}^{S}$ corresponding to subsets $Q \subset S$ satisfying (8.4). It follows from Proposition 8.1 that

$$
\max _{1 \leq i \leq n}\left\{\left|\left\langle X_{Q}, h_{H_{i}}\right\rangle\right|\right\}=\ell
$$

For each $i$, let

$$
a_{i}= \begin{cases}0, & \text { if }\left\langle X_{Q}, h_{H_{i}}\right\rangle \geq 0 \\ 1, & \text { if }\left\langle X_{Q}, h_{H_{i}}\right\rangle<0\end{cases}
$$


Then it follows from (8.13) and the computation done in Case 1) that

$$
\max _{1 \leq i \leq n}\left\{\left|\left\langle X_{Q}, h_{H_{i}}\right\rangle\right|\right\}=\ell-1 .
$$

Repeating this process $\ell-1$ times, we see that there exists (not necessarily distinct) elements $\mathbf{a}_{1}, \ldots, \mathbf{a}_{\ell} \in B_{n}$ such that

$$
X_{Q}-\sum_{1 \leq j \leq \ell} \mathbf{a}_{j} \in \operatorname{ker} H .
$$

Hence, as is argued in Case 1) above, [4, Proposition 4.9] implies the existence of a positive integer $n$, a number of four-element subsets $M_{1}, \ldots, M_{p} \subset S_{(n)}$ with $M_{i} \cap \rho\left(M_{i}\right)=\phi$, and elements $s_{1}, \ldots, s_{q} \in\left(S_{1}\right)_{(n)}$ such that

$$
\begin{aligned}
X_{Q}-\sum_{1 \leq j \leq \ell} \mathbf{a}_{j}= & \sum_{1 \leq \alpha \leq p^{\prime}} X_{M_{\alpha}}-\sum_{p^{\prime}+1 \leq \beta \leq p} X_{M_{\beta}} \\
& +\sum_{1 \leq \gamma \leq q^{\prime}} X_{\left\{s_{\gamma}, \rho s_{\gamma}\right\}}-\sum_{q^{\prime}+1 \leq \delta \leq q} X_{\left\{s_{\delta}, \rho s_{\delta}\right\}}, \\
& X_{M_{\alpha}}, X_{M_{\beta}} \in \operatorname{ker} H .
\end{aligned}
$$

Hence we have

$$
\begin{aligned}
& X_{Q}+\sum_{p^{\prime}+1 \leq \beta \leq p} X_{M_{\beta}}+\sum_{p^{\prime}+1 \leq \beta \leq p} X_{\rho\left(M_{\beta}\right)}+\sum_{q^{\prime}+1 \leq \delta \leq q} X_{\left\{s_{\delta}, \rho s_{\delta}\right\}} \\
& =\sum_{1 \leq \alpha \leq p^{\prime}} X_{M_{\alpha}}+\sum_{p^{\prime}+1 \leq \beta \leq p} X_{\rho\left(M_{\beta}\right)}+\sum_{1 \leq \gamma \leq q^{\prime}} X_{\left\{s_{\gamma}, \rho s_{\gamma}\right\}}+\sum_{1 \leq j \leq \ell} \mathbf{a}_{j} .
\end{aligned}
$$

Then by a similar reasoning to the one employed in Case 1), we see that

$$
[Q] \in F_{a}^{(k-\ell) / 2} H^{k}\left(A^{n}, \mathbf{Q}\right) .
$$

Thus the proof is completed.

Corollary (of the proof). Suppose that the Hodge cycles of codimension two on $A_{\boldsymbol{A}\left(2^{n}\right)}(G ; K)$ is algebraic. Then the whole $G H C$ holds for any self-product $A_{\boldsymbol{A}\left(2^{n}\right)}(G ; K)^{m}$.

Proof of Corollary. Take $S=S_{(m)}$ in the above proof. Then the abelian variety $A$ which corresponds to $S$ through (4.B) is the self-product $A_{\boldsymbol{A}\left(2^{n}\right)}$ $(G ; K)^{m}$, as is remarked there. Since $A_{\boldsymbol{A}\left(2^{n}\right)}(G ; K)$ is 2-dominated, the assumption of the corollary implies that the usual Hodge Conjecture holds true for $A_{\boldsymbol{A}\left(2^{n}\right)}(G ; K)^{m}$. Thus the same argument as in the proof of Theorem 8.2 shows the validity of the corollary.

Thus combining this corollary with Proposition 6.5 and Lemma 2.1, we obtain the following. 
Theorem 8.3. $\quad$ Suppose that any Hodge cycle of codimension two on $A_{\boldsymbol{A}\left(2^{n}\right)}(G ; K)$ is algebraic for any pair $(G, K)$. Then the whole $G H C$ holds for any abelian variety of CM-type.

\section{References}

[1] Dodson, B., The structure of Galois groups of CM-fields, Trans. Amer. Math. Soc., 283 (1984), 1-32.

[2] Hazama, F., Hodge cycles on Abelian varieties of CM type, Res. Act. Fac. Sci. Engrg. Tokyo Denki Univ., 5 (1983), 31-33.

[3] Hodge cycles on abelian varieties of $S_{n}$-type, J. Alg. Geom., 9 (2000), 711-753.

[4] Lang, S., Complex Multiplication, Grundlehren Math. Wiss. 255, Springer-Verlag, New York, 1983.

[5] Lewis, D., A Survey of the Hodge Conjecture, (2 ${ }^{\text {nd }}$. ed.) CRM Monog. Ser., 10, AMS, Providence, 1999.

[6] Pohlmann, H., Algebraic cycles on abelian varieties of complex multiplication type, Ann. of Math., 88 (1968), 161-180.

[7] Shimura, G., Taniyama, Y., Complex multiplication of abelian varieties and its applications to number theory, Publ. Math. Soc. Japan, No. 6, 1961.

[8] Varchenko, A. N., Bilinear form of real configuration of hyperplanes, Adv. Math., 97 (1993), 110-144. 